\title{
Relationship between Insulin Levels and Nonpsychotic Dementia: A Systematic Review and Meta-Analysis
}

\author{
Qiu-xia Pan, Xiao-juan Li, Yue-yun Liu, Fang-fang Wang, Ya-jing Hou, Qing-lai Bian, \\ Wen-qi Qiu, Zhi-yi Yan, You-ming Jiang, and Jia-xu Chen
}

\author{
School of Basic Medical Science, Beijing University of Chinese Medicine, No. 11 North Third Ring Road, Chaoyang, \\ Beijing 100029, China \\ Correspondence should be addressed to Jia-xu Chen; chenjiaxu@hotmail.com
}

Received 20 August 2017; Accepted 16 October 2017; Published 27 December 2017

Academic Editor: Fushun Wang

Copyright ( 2017 Qiu-xia Pan et al. This is an open access article distributed under the Creative Commons Attribution License, which permits unrestricted use, distribution, and reproduction in any medium, provided the original work is properly cited.

\begin{abstract}
Objectives. To explore the relationship between insulin levels and nonpsychotic dementia. Methods. Six electronic databases (PubMed, Cochrane, SCI, CNKI, VIP, and Wanfang) were searched from January 1, 2007, to March 1, 2017. Experimental or observational studies that enrolled people with nonpsychotic dementia or abnormal insulin levels in which insulin levels or MMSE scores (events in nonpsychotic dementia) were the outcome measures. Random-effects models were chosen for this meta-analysis. Sample size, mean, s.d., and events were primarily used to generate effect sizes (with the PRIMA registration number CRD42017069860). Results. 50 articles met the final inclusion criteria. Insulin levels in cerebrospinal fluid were lower (Hedges' $g=1.196,95 \% \mathrm{CI}=0.238$ to 2.514 , and $P=0.014$ ), while the levels in peripheral blood were higher in nonpsychotic dementia patients (Hedges' $g=0.853$ and $95 \% \mathrm{CI}=0.579$ to 1.127 ), and MMSE scores were significantly lower in the high insulin group than in the healthy control group (Hedges' $g=0.334,95 \% \mathrm{CI}=0.249$ to 0.419 , and $P=0.000$ ). Conclusions. Our comprehensive results indicate that blood insulin levels may increase in patients with nonpsychotic dementia.
\end{abstract}

\section{Introduction}

Dementia is a general term for a decline in mental ability severe enough to interfere with daily life, and it is a common clinical syndrome that is not only a leading cause of death globally but also a burden on families and society. There is no one test to determine if someone has dementia. Doctors diagnose Alzheimer's and other types of dementia based on a careful medical history, a physical examination, laboratory tests, and the characteristic changes in thinking, day-to-day function, and behavior associated with each type. At present, all of the Mini-Mental State Examination (MMSE), Hasegawa's dementia scale, clinical dementia rating, cognitive abilities screening instrument, Alzheimer's disease assessment scale-cognitive section, activity of living, Functional Activities Questionnaire, and clock-drawing test can be used in the diagnosis of dementia, among which, the MMSE is widely used.
As it is harder to determine the exact type of dementia because the symptoms and brain changes of different dementias can overlap, and in some cases, a doctor may diagnose "dementia" and not specify a type. In these cases, we divided the dementia into two aspects (psychotic dementia and nonpsychotic dementia) according to the day-to-day functions, behaviors, and medical history of the patients. Psychotic dementia is caused by mental illness, which means that dementia is caused by depression, schizophrenia, and other mental disorders, while nonpsychotic dementia excludes the dementia caused by mental illness (psychotic dementia) [1], for example, Alzheimer's disease (AD), vascular dementia (VD), and brain lesions. In addition, mild cognitive impairment (MCI) is a "clinical" diagnosis representing a doctor's best professional judgment about the reason for a person's symptoms. MCI causes a slight but noticeable and measurable decline in cognitive abilities, including memory and thinking skills. A person with $\mathrm{MCI}$ is at an increased risk 
of developing Alzheimer's or another dementia. Medical history, assessment of independent function and daily activities, input from a family member or trusted friend, assessment of mental status, in-office neurological examination, evaluation of mood, and laboratory tests were the primary diagnostic route.

Many risk factors are associated with nonpsychotic dementia. These include older age, gender (i.e., female), a low educational level, a low socioeconomic level, certain physical diseases, and a family genetic history. Recently, abnormal insulin levels were shown to be an important factor that influences the occurrence of nonpsychotic dementia. Umegaki et al. [2] performed a prospective study in which cognitive function scores were assessed at baseline and after 3 years in the same patient group. The results suggested that higher insulin and glycol-hemoglobin levels were associated with diabetes-related cognitive dysfunction.

Insulin is an active substance with a variety of biological functions that affect growth and apoptosis in neurons by participating in blood glucose metabolism [3-8]. The discovery of insulin in the brain suggested that insulin not only participates in metabolism and growth but is also involved in higher cognitive functions, such as learning and memory [3-5]. Insulin has been shown to increase the expression of amyloid precursor protein (APP), betaamyloid 42 (A $\beta 42)$, and hyperphosphorylated tau in the hippocampus and frontal cortex in rats $[6,9]$. Thus, there may be mechanistic and sequential associations among insulin, impaired cognitive function, and structural ADlike changes.

In humans, aging is associated with decreased metabolic turnover, decreased glucose utilization, and decreased insulin levels because of effects on the regulation of the insulin signaling pathway. Some authors have argued that abnormal insulin metabolism is caused by aging and does not share a causal relationship with nonpsychotic dementia. For example, Burns et al. [8] assessed the relationship between insulin resistance and conditions including cognitive decline and brain atrophy for two years in patients with early $\mathrm{AD}$ and nondemented controls. The authors found that insulin was differentially associated with cognitive decline and atrophy in $\mathrm{AD}$ and elderly patients. Furthermore, higher levels of peripheral insulin may exert $\mathrm{AD}$-specific benefits, and insulin signaling may be affected by $\mathrm{AD}$-associated systemic physiological changes. Finally, Dorrance et al. [10] supported the notion that insulin resistance exerts positive effects on cerebral vasculature dementia.

Furthermore, exogenous insulin interventions or treatments have been shown to alleviate nonpsychotic dementia symptoms in patients with insulin resistance and improve their Mini-Mental State Examination (MMSE) scores $[11,12]$. However, other studies have demonstrated that insulin levels are negatively correlated with nonpsychotic dementia [13]. In addition, some studies have found no relationship between the occurrence of nonpsychotic dementia and insulin levels when patients were compared with healthy control subjects (HCs) [14, 15]. Therefore, both negative and positive correlations have been observed, resulting in controversy regarding whether high insulin levels or low insulin levels cause nonpsychotic dementia. Thus, a meta-analysis of this subject is warranted.

The present meta-analysis was aimed at determining whether nonpsychotic dementia is associated with altered levels of insulin in the blood or cerebrospinal fluid (CSF). We used meta-analytical techniques because they allow data from individual studies to be quantitatively combined to improve the strength of preclinical and clinical evidence.

\section{Materials and Methods}

The meta-analyses performed in this study adhered to the guidelines recommended by the Preferred Reporting Items for Systematic Reviews and Meta-Analysis (PRISMA) statement [16] and are registered with the international prospective register of systematic reviews (PROSPERO). Its PRISMA registration number is CRD42017069860.

2.1. Literature Search. Two investigators (Qiu-xia Pan and Xiao-juan Li) independently performed a systematic review of English language publications using the PubMed, SCI, and Cochrane Library databases and Chinese language publications using the CNKI, VIP, and Wanfang databases.

The following search terms were used in the database search: (Dementia or Alzheimer's disease/AD or Mild cognitive impairment/MCI) and (Insulin or insulin signaling pathway). The search was performed to obtain articles published from January 1, 2007, to March 1, 2017. The initial search generated 1287 records, including 474 English and 813 Chinese records. After we screened the titles and abstracts, 263 appropriate articles, including 120 English and 143 Chinese papers, that were related to the present subject were selected for full-text scrutiny. Original studies that reported data on peripheral blood and CSF insulin concentrations in at least two groups of subjects (i.e., dementia or $\mathrm{AD}$ and $\mathrm{MCI}$ ) were included.

2.2. Quality Assessment. Studies were appraised for methodological quality using the Newcastle-Ottawa Scale (NOS). The NOS defines the study quality of observational studies (i.e., case-control studies and cross-sectional studies) using an 8-item scale (scored 0-9 points). Two investigators (Qiu-xia Pan and Xiao-juan Li) independently assessed the quality of each paper, and any disagreements were resolved by consensus in a group meeting.

2.3. Study Selection. After further evaluating the 135 originally selected articles, 50 high-quality (i.e., had 5-9 points on the NOS, Table 1) [17] articles describing observational studies were included in this study. The remaining 85 articles were excluded for the following reasons: there were no necessary data on insulin concentrations [13, 18-27]; related genes and insulin signaling pathway proteins were the main study outcomes [28-36]; there was no clear description or record of a cognitive function evaluation [37-42]; the design of the experiments was unreasonable for the purpose of this study [43-52]; the experimental methods were incomplete [53-60]; the papers did not provide completely related data [61-66]; data from only one group were reported $[2,67-74]$; the papers reported data from the same cohort of 
TABLE 1: Quality assessment of the included studies according to the Newcastle-Ottawa Scale (NOS)*.

\begin{tabular}{|c|c|c|c|c|c|c|c|c|c|c|c|}
\hline Study names & S1 & S2 & S3 & S4 & Cla & $\mathrm{Clb}$ & E1a & E1b & $\mathrm{E} 2$ & E3 & Total points \\
\hline Si-ling Liu, 2011 & 1 & 1 & 1 & 1 & 0 & 1 & 1 & 0 & 0 & 0 & 6 \\
\hline Qin-yun Li, 2015 & 1 & 1 & 1 & 1 & 0 & 1 & 1 & 0 & 1 & 0 & 7 \\
\hline Zong-yuan Wang, 2008 & 1 & 1 & 1 & 1 & 0 & 1 & 1 & 0 & 1 & 0 & 7 \\
\hline Abimbola A. Akintola, 2015 & 0 & 1 & 1 & 0 & 0 & 1 & 1 & 0 & 1 & 0 & 5 \\
\hline Angela J. Hanson, 2016 & 1 & 1 & 0 & 0 & 1 & 0 & 1 & 0 & 1 & 0 & 5 \\
\hline J.K. Morris, 2016 & 1 & 1 & 0 & 0 & 0 & 1 & 1 & 0 & 1 & 0 & 5 \\
\hline G. Stennis Watson, 2009 & 1 & 1 & 1 & 1 & 0 & 1 & 1 & 0 & 1 & 0 & 7 \\
\hline Robert Krikorian, 2012 & 1 & 1 & 0 & 0 & 0 & 1 & 1 & 0 & 1 & 0 & 5 \\
\hline E. Rönnemaa, B. Zethelius, and J. Sundelöf, 2009 & 1 & 1 & 0 & 1 & 1 & 0 & 0 & 1 & 1 & 0 & 6 \\
\hline Galit Weinstein, 2015 & 0 & 1 & 1 & 1 & 0 & 1 & 1 & 0 & 1 & 0 & 6 \\
\hline Jeffrey M. Burns, 2012 & 0 & 1 & 1 & 1 & 0 & 1 & 1 & 0 & 1 & 0 & 6 \\
\hline Auriel A. Willette, 2015 & 0 & 1 & 1 & 1 & 0 & 1 & 1 & 0 & 1 & 0 & 6 \\
\hline Amber S. Watts, 2013 & 1 & 1 & 1 & 1 & 1 & 1 & 1 & 0 & 1 & 1 & 9 \\
\hline Josh D. Woolley, 2014 & 1 & 1 & 0 & 0 & 1 & 0 & 1 & 0 & 1 & 1 & 6 \\
\hline Xiao-hong Sun, 2010 & 1 & 1 & 0 & 1 & 1 & 0 & 1 & 0 & 1 & 0 & 6 \\
\hline Lu Zhang, 2011 & 1 & 1 & 0 & 1 & 1 & 0 & 1 & 0 & 1 & 0 & 6 \\
\hline Wei-gang Liu, 2009 & 1 & 1 & 0 & 0 & 1 & 0 & 1 & 0 & 1 & 0 & 5 \\
\hline Gui-qing Chen, 2015 & 1 & 1 & 0 & 1 & 1 & 0 & 1 & 0 & 1 & 1 & 7 \\
\hline Zhi-juan Wang, 2014a & 1 & 1 & 0 & 1 & 1 & 0 & 1 & 0 & 1 & 0 & 6 \\
\hline Jun-shi Zhang, 2016 & 1 & 0 & 0 & 1 & 1 & 1 & 1 & 0 & 1 & 1 & 7 \\
\hline Jin-geng Li, 2013 & 1 & 1 & 0 & 1 & 1 & 1 & 1 & 0 & 1 & 1 & 8 \\
\hline Zhi-dong Yang, 2007 & 1 & 0 & 1 & 1 & 0 & 1 & 1 & 0 & 1 & 0 & 6 \\
\hline Hong-mei Yue, 2014 & 1 & 1 & 0 & 1 & 1 & 1 & 1 & 0 & 1 & 0 & 7 \\
\hline Liang-mi Li, 2015 & 1 & 1 & 1 & 1 & 1 & 1 & 1 & 0 & 1 & 0 & 8 \\
\hline Qing-chun Xia, 2015 & 1 & 1 & 0 & 1 & 1 & 1 & 1 & 1 & 1 & 0 & 8 \\
\hline Ran Song, 2015 & 1 & 1 & 0 & 1 & 1 & 1 & 1 & 0 & 1 & 1 & 8 \\
\hline Yu-mei Yang, 2009 & 1 & 0 & 0 & 1 & 1 & 1 & 1 & 0 & 1 & 0 & 6 \\
\hline Xiao-lan Liu, 2008 & 1 & 1 & 0 & 1 & 1 & 1 & 1 & 0 & 1 & 1 & 8 \\
\hline Rong-wei Zhang, 2008 & 1 & 1 & 0 & 0 & 1 & 0 & 1 & 0 & 1 & 0 & 5 \\
\hline Bin-bin Zang, 2011 & 1 & 1 & 0 & 1 & 1 & 1 & 1 & 0 & 1 & 0 & 7 \\
\hline Zhi-juan Wang, 2014b & 1 & 1 & 1 & 1 & 1 & 0 & 1 & 0 & 1 & 0 & 7 \\
\hline Hong-shan Pan, 2016 & 1 & 1 & 0 & 1 & 1 & 0 & 1 & 0 & 1 & 0 & 6 \\
\hline Nan Mu, 2010 & 1 & 1 & 1 & 1 & 1 & 0 & 1 & 0 & 1 & 0 & 7 \\
\hline Yan-Wu, 2008 & 1 & 1 & 0 & 1 & 1 & 0 & 1 & 0 & 1 & 0 & 6 \\
\hline Hong-li Li, 2013 & 1 & 1 & 0 & 1 & 1 & 0 & 1 & 0 & 1 & 0 & 6 \\
\hline Jill K. Morris, 2014 & 1 & 0 & 0 & 0 & 1 & 1 & 1 & 0 & 1 & 1 & 6 \\
\hline Wen-qing Xia, 2016 & 1 & 1 & 1 & 1 & 1 & 0 & 1 & 0 & 1 & 0 & 7 \\
\hline Chin-Chou Huang, 2014 & 1 & 1 & 1 & 1 & 0 & 1 & 1 & 0 & 1 & 0 & 7 \\
\hline Rosebud O. Roberts, 2014 & 1 & 1 & 1 & 0 & 0 & 1 & 1 & 0 & 1 & 0 & 6 \\
\hline Mkaya Mwamburi, 2016* & 1 & 1 & 0 & 1 & 0 & 0 & 1 & 1 & 1 & 0 & 6 \\
\hline Heather Kenna, 2013 & 1 & 1 & 1 & 1 & 1 & 0 & 1 & 0 & 1 & 0 & 7 \\
\hline Yen-Chun Fan, 2017 & 1 & 1 & 1 & 1 & 0 & 1 & 1 & 0 & 1 & 0 & 7 \\
\hline Laura D. Baker, 2011 & 0 & 1 & 1 & 1 & 0 & 1 & 1 & 0 & 1 & 0 & 6 \\
\hline M.S. Beeri, 2008 & 0 & 1 & 1 & 1 & 0 & 1 & 1 & 0 & 1 & 0 & 6 \\
\hline Hannah Bruehl, 2009 & 1 & 1 & 1 & 1 & 1 & 1 & 1 & 1 & 1 & 0 & 9 \\
\hline Xiao-bing Zhou, 2012 & 0 & 1 & 0 & 1 & 1 & 0 & 1 & 0 & 1 & 0 & 5 \\
\hline Qiong-yu Zhang, 2012 & 1 & 1 & 0 & 0 & 1 & 1 & 1 & 0 & 1 & 0 & 6 \\
\hline
\end{tabular}


TABLE 1: Continued.

\begin{tabular}{lccccccccccc}
\hline Study names & S1 & S2 & S3 & S4 & C1a & C1b & E1a & E1b & E2 & E3 & Total points \\
\hline Xiao-hong, Zhao, 2009 & 1 & 1 & 0 & 1 & 1 & 1 & 1 & 0 & 1 & 0 & 7 \\
Jun-yi Wu, 2013 & 1 & 1 & 0 & 1 & 1 & 1 & 1 & 0 & 1 & 0 & 7 \\
Sheng Huang, 2015 & 1 & 1 & 0 & 1 & 1 & 1 & 1 & 0 & 1 & 0 & 7 \\
\hline
\end{tabular}

Note. S1: eligibility criteria; S2: representativeness of the cases; S3: community controls; S4: the controls had no history of disease (endpoint); C1a: important factor basis between two groups; C1b: study controls for additional factor basis between two groups; E1a: secure record of exposure; E1b: structured interview where blind to case about the exposure; E2: same method of ascertainment for cases and controls; E3: nonresponse rate. ${ }^{*}$ Cross-sectional study.

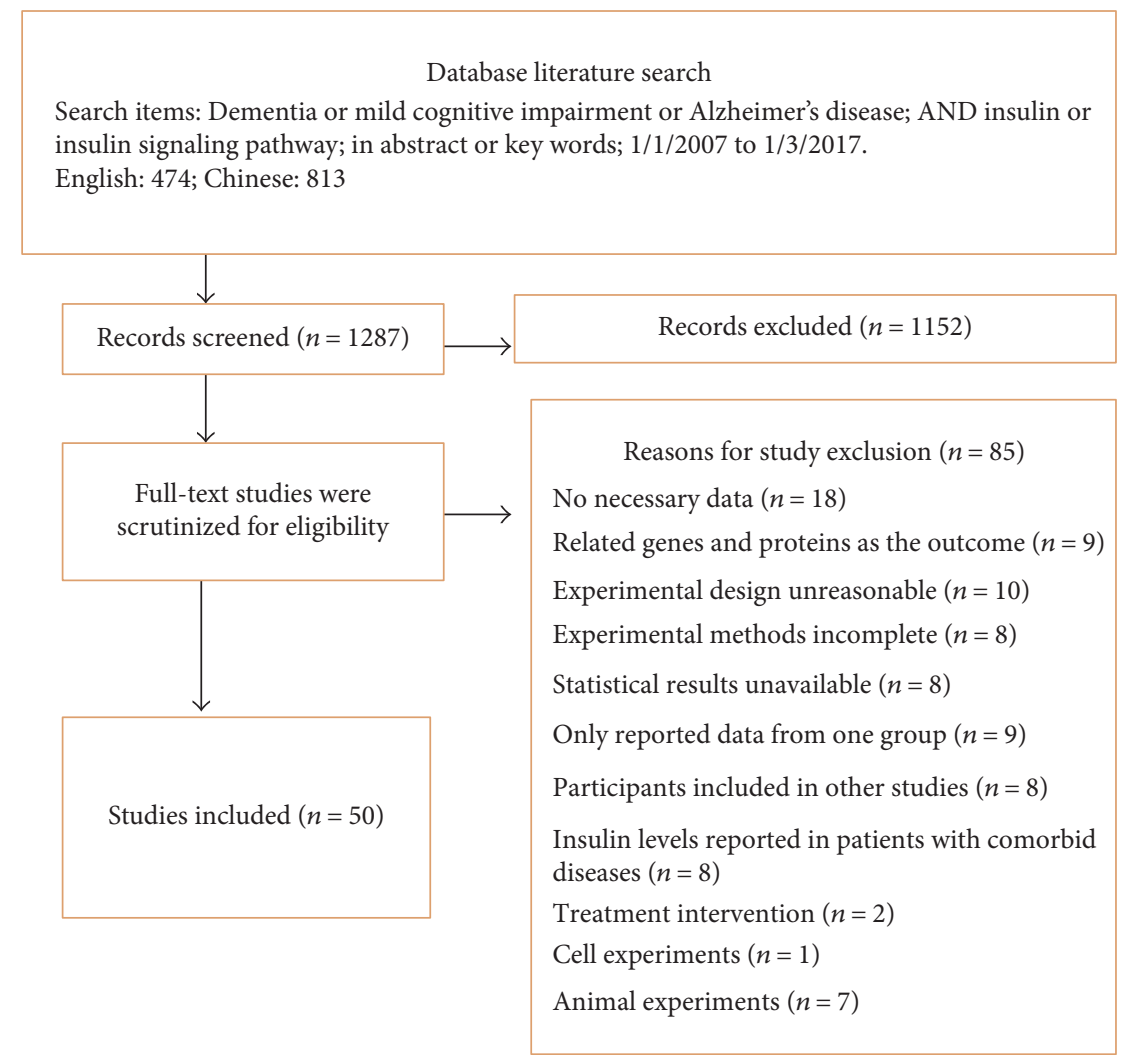

Figure 1: Preferred Reporting Items for Systematic Reviews and Meta-Analysis flowchart of the literature search.

patients who were used in another study [75-81]; insulin levels were reported in patients with comorbid diseases (in addition to diabetes, metabolic syndrome and related diseases can also lead to nonpsychotic dementia [82-89]), such as cognitive dysfunction caused by depression or anxiety [85]; serum insulin was measured before and after insulin treatment or other treatments that could influence insulin metabolism in patients with nonpsychotic dementia; only changes in the MMSE scores before and after the treatment were analyzed, while differences between the observation group and the control group were not analyzed $[11,12]$; and the studies involved cell experiments [90] or animal experiments $[6,91-96]$ (for the flowchart, see Figure 1).

2.4. Data Extraction. Data regarding sample size, mean, standard deviation (s.d.), events, and $P$ values were extracted as the primary outcomes. Two investigators (Qiu-xia Pan and Xiao-juan $\mathrm{Li}$ ) independently extracted the data, and the results were verified by another team member (Zhi-yi Yan). Any inconsistencies were resolved by consensus. Tables 2 and 3 summarized the included studies and present the demographic and clinical characteristics of the included patients.

2.5. Statistical Analysis. Comprehensive Meta-Analysis Version 2 software (Biostat, Englewood, NJ, USA) was used for all statistical analyses. The sample size, mean, s.d., and events were primarily used to generate effect sizes (ESs) (the sample size and $P$ value were used in some studies in which the mean, s.d., and events were not available). The ESs were calculated as the standardized mean difference in insulin levels or MMSE scores between groups and converted to Hedges' $g$, which provides an unbiased ES that is adjusted for the sample size. In pooled ESs, $95 \%$ confidence intervals (95\% CIs) were used to assess significant differences. Random-effects models were chosen for this metaanalysis because we hypothesized that within-study and 


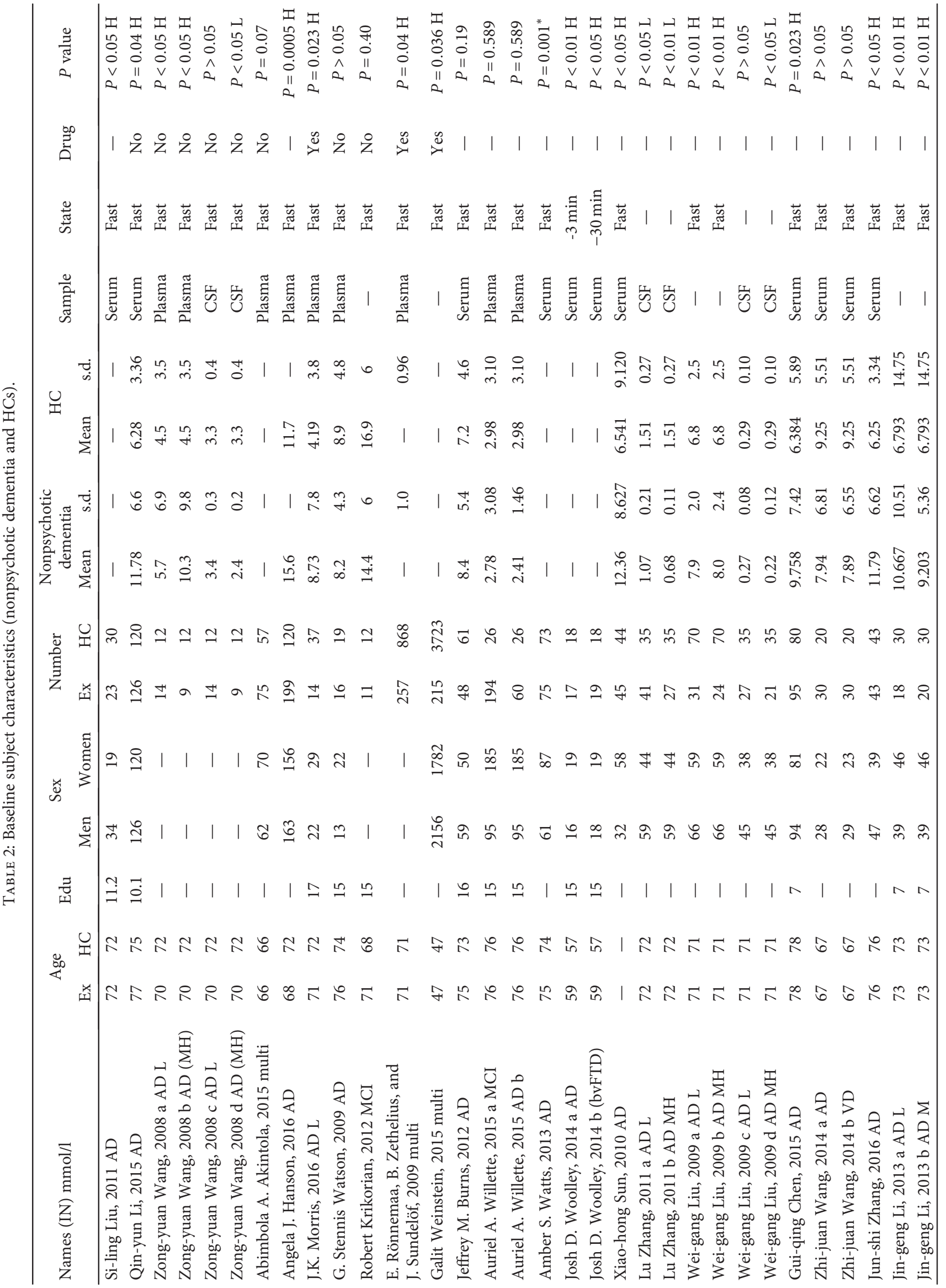




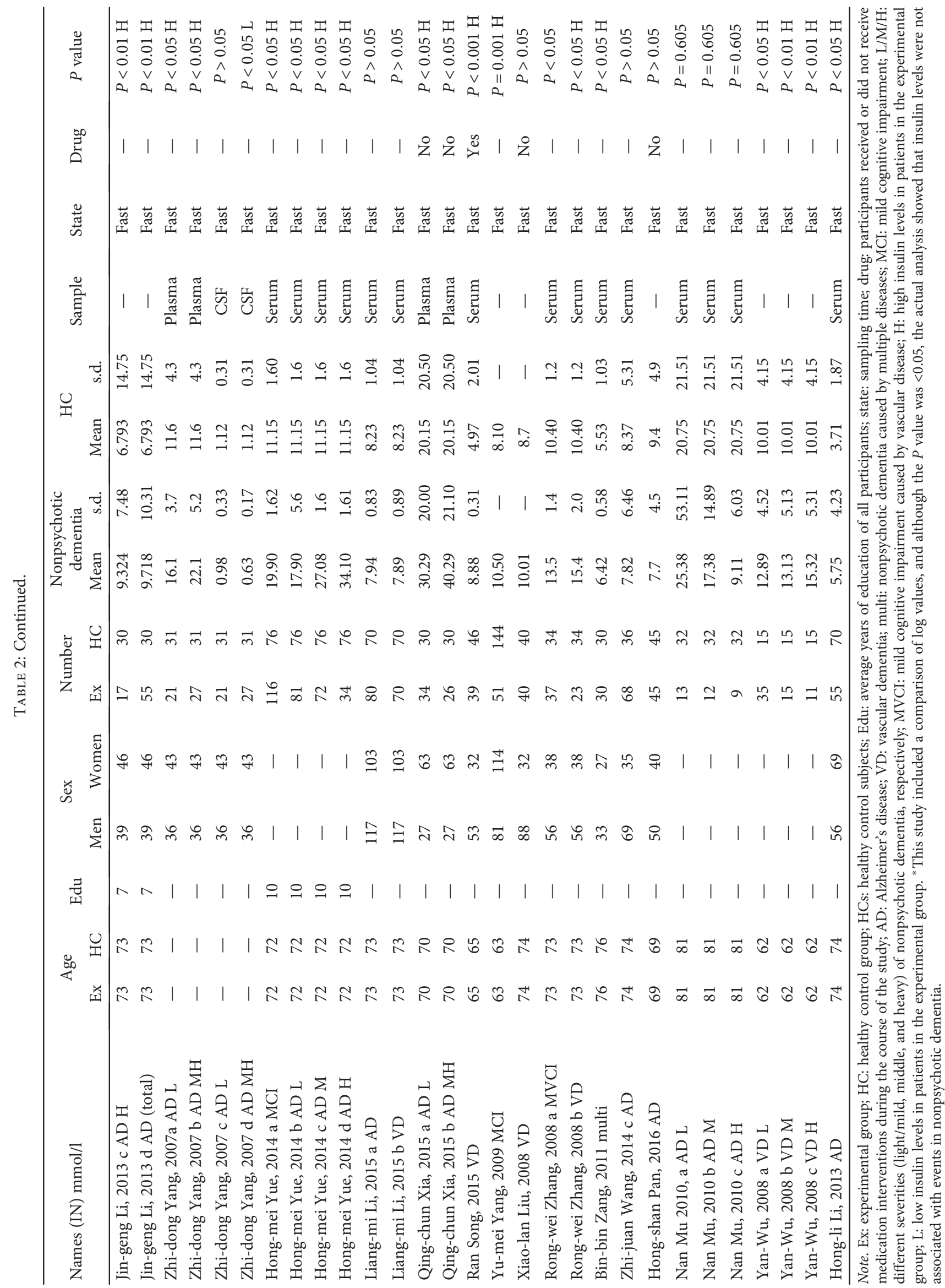


TABLE 3: Baseline characteristics of the subjects (abnormal insulin levels and HCs).

\begin{tabular}{lccccccccccccc}
\hline \multirow{2}{*}{ Names (events/MMSEs) } & \multicolumn{3}{c}{ Age } & \multicolumn{1}{c}{ Ex } & HC & Edu & Men & Women & Ex & HC & $\begin{array}{c}\text { Mean } \\
\text { Events }\end{array}$ & $\begin{array}{c}\text { Mean } \\
\text { Events }\end{array}$ & $P$ value \\
\hline Wen-qing Xia, 2016 & 59.5 & 56.2 & 10.3 & 32 & 38 & 38 & 32 & 28.8 & 1.1 & 29.1 & 1.2 & $P<0.001 \mathrm{H}$ \\
Chin-Chou Huang, 2014 & 79 & 79 & - & 73941 & 68803 & 71433 & 71311 & 8572 & 4992 & $P<0.001 \mathrm{H}$ \\
Rosebud O. Roberts, 2014 & 80 & 79 & 14 & 423 & 326 & 154 & 595 & - & - & - & - & $P=0.914$ \\
Mkaya Mwamburi, 2016 a & 75 & 80 & 12 & - & - & 70 & 67 & 25.3 & 3.4 & 25.4 & 4.2 & $P=0.914$ \\
Mkaya Mwamburi, 2016 b & 75 & 80 & 12 & - & - & 67 & 67 & 25.1 & 3.6 & 25.4 & 4.2 & $P=0.914$ \\
Mkaya Mwamburi, 2016 c & 75 & 80 & 12 & - & - & 83 & 67 & 25.4 & 3.2 & 25.4 & 4.2 & $P>0.05$ \\
Heather Kenna, 2013 & 59 & 57 & 16 & - & - & 10 & 10 & 28.9 & 0.9 & 29.5 & 1.0 & $P<0.001 \mathrm{H}$ \\
Yen-Chun Fan, 2017 & 53 & 53 & - & 27360 & 24220 & 10316 & 41246 & 413 & 825 & $P<0.05 \mathrm{H}$ \\
Laura D. Baker, 2011 & 74 & 74 & - & - & - & 12 & 11 & - & 3.3 & - & 1.5 & $P<0.05 \mathrm{H}$ \\
M.S. Beeri, 2008 & - & - & - & 111 & 137 & 124 & 124 & - & - & - & - & $P<0.05 \mathrm{H}$ \\
Hannah Bruehl, 2009 & 59 & 60 & 15 & 42 & 46 & 41 & 47 & - & - & - & - & $--\mathrm{H}$ \\
Xiao-bing Zhou, 2012 & 73 & 73 & - & 252 & 280 & 162 & 370 & 81 & 66 & $P<0.05 \mathrm{H}$ \\
Qiong-yu Zhang, 2012 & 62 & 62 & 9 & - & - & 81 & 103 & 25.34 & 4.72 & 23.42 & 5.50 & $P<0.05 \mathrm{H}$ \\
Xiao-hong Zhao, 2009 & 65 & 65 & - & - & - & 31 & 71 & 17.46 & 9.51 & 10.19 & 6.09 & $P<0.001 \mathrm{H}$ \\
Jun-yi Wu, 2013 & 71 & 71 & - & 93 & 48 & 60 & 81 & 38 & & 25 & $P<0.05$ \\
Sheng Huang, 2015 & 65 & 65 & - & 49 & 51 & 58 & 42 & 41 & & 18 & $P<0.05$ \\
Jill K. Morris, 2014 & 76 & 74 & 16 & 165 & 99 & 97 & 167 & - & & - & $P=0.009 \mathrm{H}$ \\
\hline
\end{tabular}

Note. Ex: experimental group; HC: healthy control group; HCs: healthy control subjects; Edu: average years of education of all participants; H: high insulin levels in patients in the experimental group; L: low insulin levels in patients in the experimental group.

between-study moderators would result in differences in the true ESs [97]. We excluded one study at a time to determine whether the results were unduly secondary to a particular study. Data regarding the average age and gender distribution of the patients and the classification and severity of cases of nonpsychotic dementia (i.e., MMSE) were also extracted.

Significant differences in heterogeneity across studies were assessed using Cochran's $Q$ test [98], and statistical significance was set at $P<0.1$. Using these parameters, we found that there was between-study heterogeneity. Inconsistencies across studies were identified using the $I^{2}$ index, which evaluates the impact of heterogeneity. $I^{2}$ values of $0.25,0.50$, and 0.75 indicate small, moderate, and high levels of heterogeneity, respectively. We then performed unrestricted maximum-likelihood random-effects metaregressions of the ESs [99] to determine whether covariates, including age, gender distribution (i.e., the proportion of males) and MMSE scores, represented moderators that influenced the ESs. We then performed unrestricted maximumlikelihood random-effects metaregressions of the ESs.

Funnel plots were generated by plotting the ESs against the precision (inverse of the standard error) of each study and used to visually inspect the studies for publication bias. The significance of any observed publication bias was determined using Egger's test [100], which assesses the degree of asymmetry in funnel plots. The classic failsafe $N$ method [101], which is an analysis that results in the number of missing (unpublished) studies that would increase the observed $P$ value to $>0.05$, was also used to investigate publication bias. Statistical significance was set at a $P$ value $<0.05$ unless otherwise indicated. $P$ values $<0.1$ were reported as trends.

\section{Results}

3.1. Included Studies. In total, 50 high-quality studies (5-9 points) were included in this analysis. These included 206207 participants, and 77 sets of data were summarized for the subsequent analysis. The results were divided into the following three areas according to differences in study objectives: (1) the relationship between insulin levels in the CSF and the risk of nonpsychotic dementia; (2) variability in cognitive function scores (i.e., MMSE scores) and insulin levels in the peripheral blood; and (3) differences in insulin levels between nonpsychotic dementia and nondementia patients (assuming that high insulin levels and nonpsychotic dementia are positively correlated).

3.2. Main Associations between CSF Insulin Levels and Nonpsychotic Dementia. First, we compared the insulin levels $(\mathrm{mU} / \mathrm{L})$ in the CSF between patients with nonpsychotic dementia and HCs. Eight sets of data were extracted from 4 studies involving a total of 300 individuals. A randomeffects meta-analysis was performed, and the results showed that the nonpsychotic dementia patients had significantly lower CSF insulin levels than were observed in the HCs (Hedges' $g=1.196,95 \% \mathrm{CI}=0.238$ to 2.514 , and $P=0.014$; Figure 2). A sensitivity analysis indicated that our results were significantly influenced by all studies (Additional File 1). In addition, significant heterogeneity 
Meta-analysis

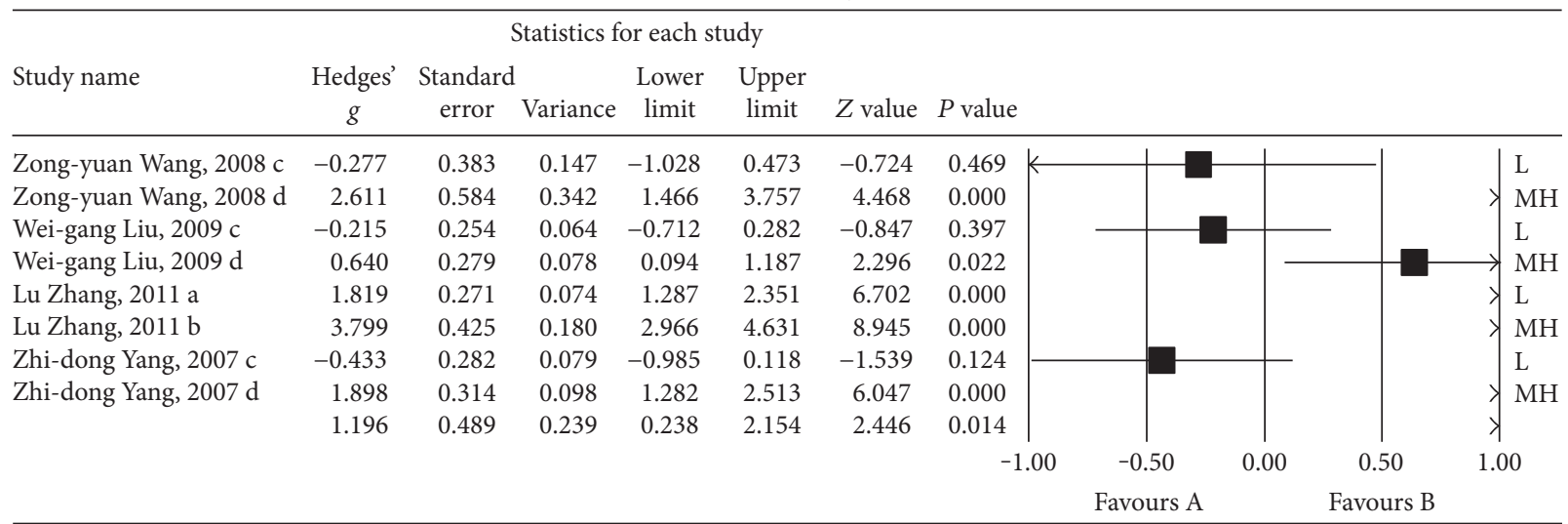

FIGURE 2: Forest plot of the random-effects meta-analysis of differences in CSF insulin levels between nonpsychotic dementia patients and healthy controls (HC). In all, 8 sets of data encompassing a total of 300 individuals were included. The sizes of the squares are proportional to study weight. CI, confidence interval.

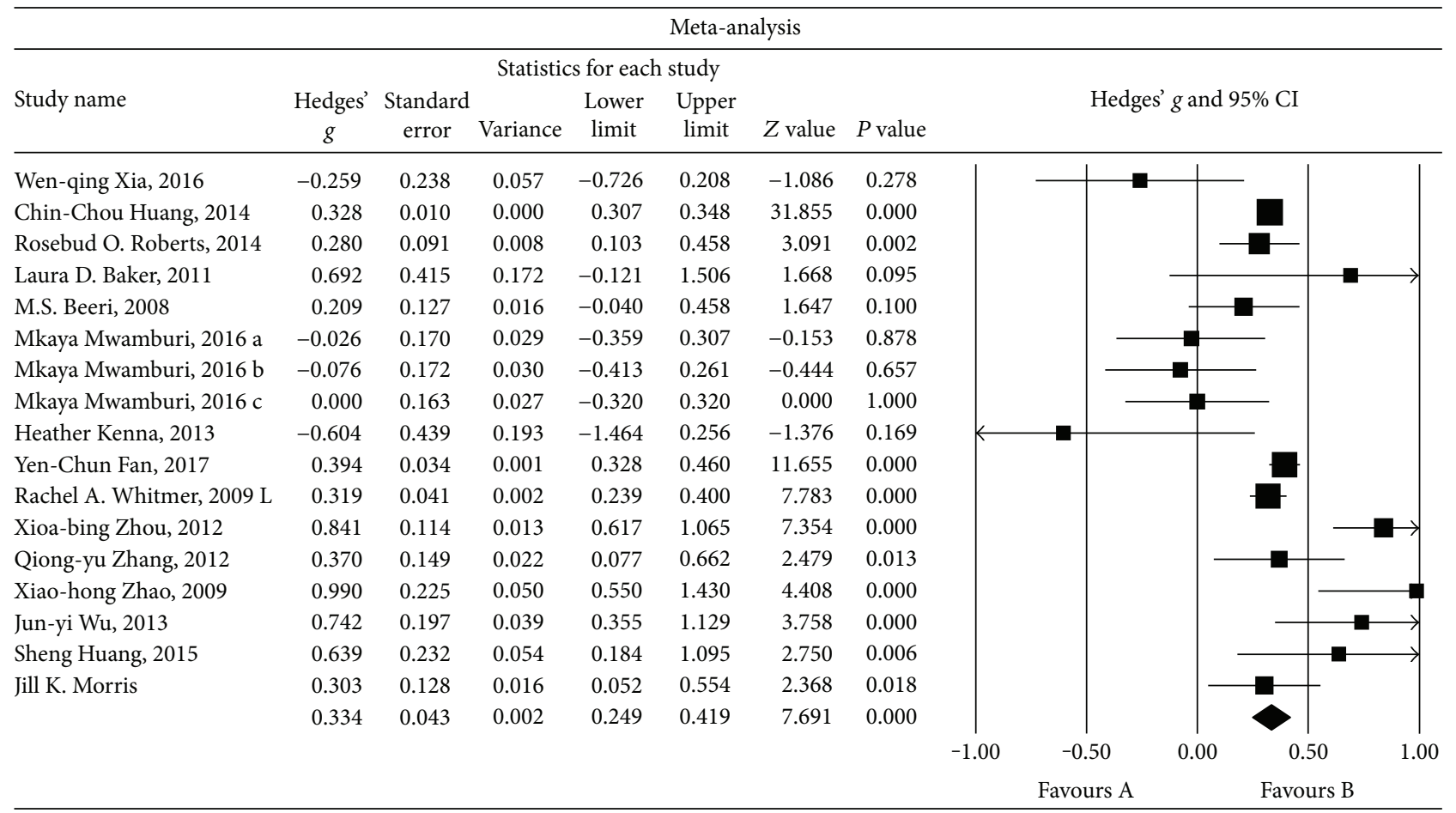

FIGURE 3: Forest plot of the random-effects meta-analysis of differences in MMSE scores between abnormal insulin levels and HC subjects. In all, 17 sets of data encompassing a total of 197114 individuals were included. The sizes of the squares are proportional to study weight. CI, confidence interval.

was observed among the studies in this meta-analysis $(Q=$ 128.753, d.f. $=7, I^{2}=94.563$, and $\left.P=0.000\right)$.

\subsection{Main Associations between MMSE Scores and Abnormal} Insulin Levels in the Peripheral Blood. Second, we compared the MMSE scores and the incidence of nonpsychotic dementia between patients with abnormal levels of insulin and HCs. Sixteen sets of data were extracted from 15 studies involving a total of 197114 individuals. A random-effects meta-analysis was performed, and the results showed that high insulin levels were associated with a higher risk of nonpsychotic dementia than was observed in the HCs (Hedges' $g=0.334$, $95 \% \mathrm{CI}=0.249$ to 0.419 , and $P=0.000$; Figure 3 ). A sensitivity analysis indicated that our results were not unduly influenced by any particular study (Additional File 2). Furthermore, high heterogeneity was observed among the studies in this meta-analysis $\left(Q=65.130\right.$, d.f. $=16, I^{2}=$ 75.434, and $P=0.000$ ).

3.4. Main Associations between Insulin Levels and Nonpsychotic Dementia. Third, we compared the levels of insulin $(\mathrm{mU} / \mathrm{L})$ in the peripheral blood between patients with 
Meta-analysis

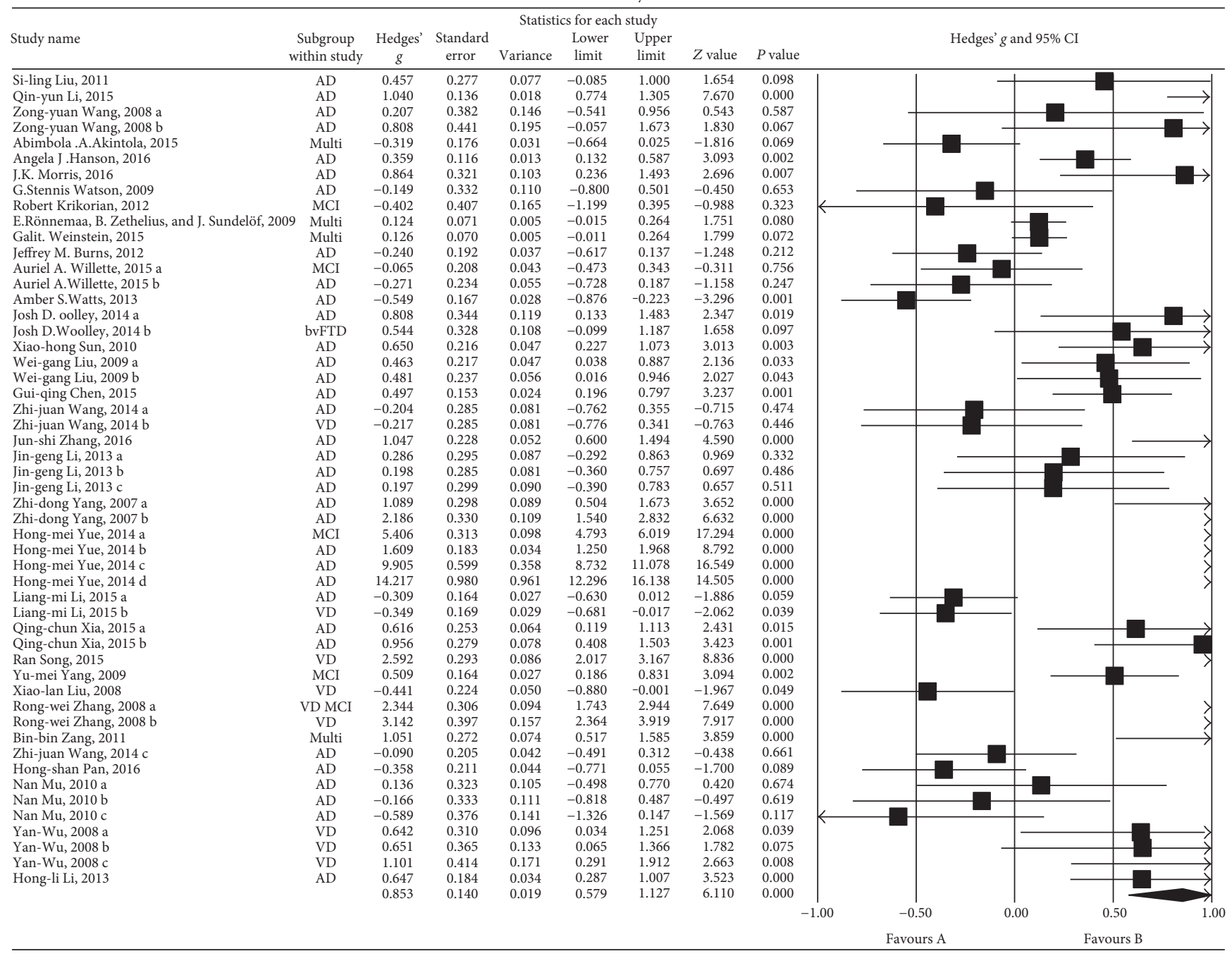

FIGURE 4: Forest plot of the random-effects meta-analysis of differences in blood insulin concentrations between nonpsychotic dementia patients and HCs. In all, 52 sets of data encompassing a total of 8931 individuals were included. The sizes of the squares are proportional to study weight. CI, confidence interval.

nonpsychotic dementia and HCs. Fifty-two sets of data were extracted from 36 studies involving a total of 8931 individuals. A random-effects meta-analysis was performed, and the results showed that the nonpsychotic dementia patients had significantly higher insulin levels than were observed in the HCs (Hedges' $g=0.853,95 \%$ $\mathrm{CI}=0.579$ to 1.127 , and $P=0.000$; Figure 4$)$. A sensitivity analysis indicated that our results were not unduly influenced by any particular study (Additional File 3). However, significant heterogeneity was observed among the studies in this meta-analysis $\left(Q=1184.942\right.$, d.f. $=51, I^{2}=95.696$, and $P=0.000)$.

3.5. Investigation of Heterogeneity. Fourth, to identify the potential sources of the heterogeneity observed in this meta-analysis, we performed subgroup analyses primarily by considering the sources of the samples (i.e., serum or plasma) and the reported medications used in the patients. We then performed subgroup analyses according to the class of the samples (i.e., VD or AD) and the degree of nonpsychotic dementia in the patients.

The subgroup analysis showed that compared with the levels observed in the HCs, serum insulin levels were significantly higher in the patients with nonpsychotic dementia (25 sets of data were extracted from 17 studies; Hedges' $g=1.482,95 \% \mathrm{CI}=0.909$ to 2.056 , and $P=0.000)$, and the same trend was observed in the plasma (13 sets of data were extracted from 9 studies; Hedges' $g=0.445$, 95\% $\mathrm{CI}=0.154$ to 0.736 , and $P=0.003$ ). Compared with the plasma group, the serum group had significantly greater ESs in the nonpsychotic dementia patients with higher insulin levels. High levels of heterogeneity were observed among studies that included serum $\left(Q=1011.719\right.$, d.f. $=25, I^{2}=$ 97.529, and $P=0.000)$ or plasma $(Q=79.543$, d.f. $=12$, $I^{2}=84.914$, and $\left.P=0.000\right)$ insulin levels. Subgroup analyses of studies involved patients who did not take any drugs, who took drugs without effect on glucose and lipid metabolism, and who are on drug withdrawal that lasted longer than 


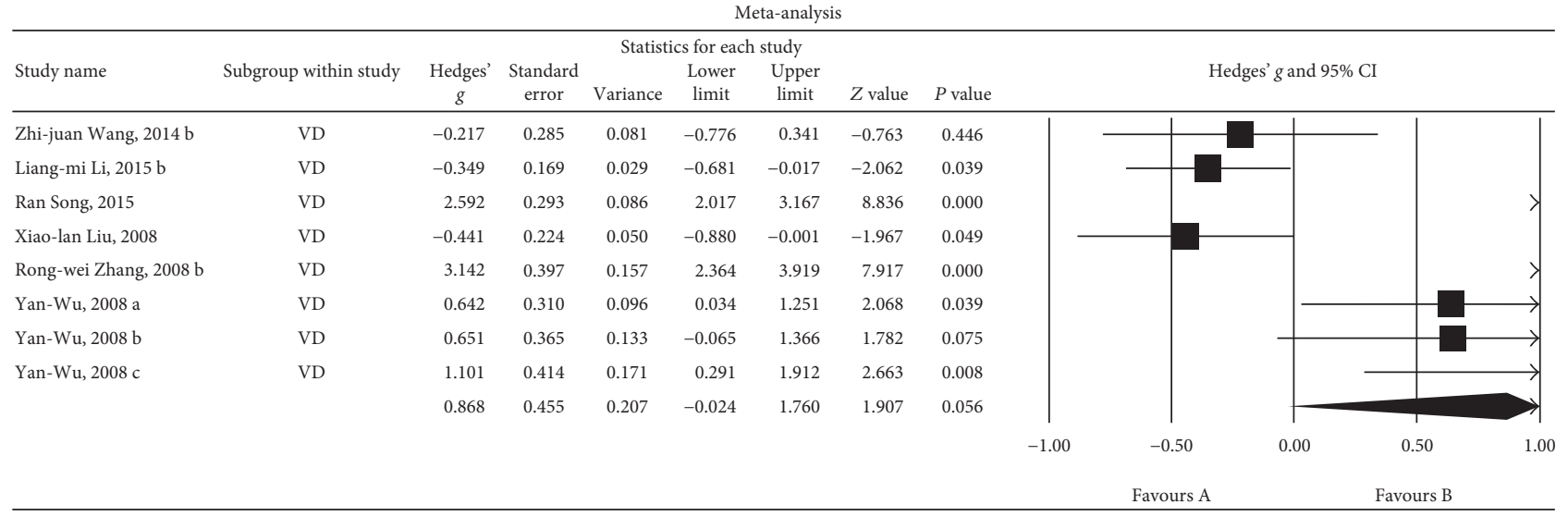

FIGURE 5: Forest plot of the random-effects meta-analysis of differences in blood insulin concentrations between VD patients and HC subjects. In all, 8 sets of data encompassing a total of 488 individuals were included. The sizes of the squares are proportional to study weight. CI, confidence interval.

2 weeks. The subjects were then grouped, and 10 sets of data were extracted from 8 studies involving a total of 731 individuals. A random-effects meta-analysis showed that insulin levels were not significantly different between the nonpsychotic dementia patients and HCs (Hedges' $g=0.194,95 \%$ $\mathrm{CI}=-0.251$ to 0.640 , and $P=0.393$ ). High levels of heterogeneity were also found $\left(Q=76.093\right.$, d.f. $=9, I^{2}=88.172$, and $P=0.000)$.

\subsection{Subgroup Analyses}

3.6.1. Associations between Insulin Levels and the VD Group. Eight sets of data were extracted from 6 studies involving a total of 488 individuals. A random-effects meta-analysis was performed, and the results showed that the risk of VD was higher in patients with high insulin levels than in the HCs (Hedges' $g=0.868,95 \% \mathrm{CI}=-0.024$ to 1.760 , and $P=0.056$; Figure 5). A sensitivity analysis indicated that two studies could have influenced this outcome (Additional File 4). This was not surprising because the $P$ values of the meta-analysis outcomes were only slightly higher than 0.05. Significant heterogeneity was observed among the studies in this meta-analysis $(Q=145.849$, d.f. $=8, I^{2}=95.201$, and $\left.P=0.000\right)$.

3.6.2. Associations between Insulin Levels and the AD Group. Thirty-four sets of data were extracted from 24 studies involving a total of 8407 individuals. A random-effects meta-analysis was performed, and the results showed that the nonpsychotic dementia patients had significantly higher insulin levels than were observed in the HCs (Hedges' $g=0.852,95 \% \mathrm{CI}=0.494$ to 1.209 , and $P=0.000$; Figure 6). A sensitivity analysis indicated that these results were not unduly influenced by any particular study (Additional File 5). However, high levels of heterogeneity among the studies were observed in this meta-analysis $(Q=678.926$, d.f. $=33, I^{2}=95.139$, and $\left.P=0.000\right)$.

Fewer than 5 studies focused on the relationship between abnormal insulin levels and nonpsychotic dementia types other than $\mathrm{AD}$ and $\mathrm{VD}$. Hence, we compared only the relationships between abnormal insulin levels and $\mathrm{AD}$ or VD because these two subgroups showed high heterogeneity. Thus, we next sought to determine whether there are differences in blood insulin levels between HCs and patients with MCI, mild (or light) nonpsychotic dementia (L, MMSE scores $\geq 16$ ) and moderate or heavy nonpsychotic dementia $(\mathrm{MH}, \mathrm{MMSE}$ scores $<16)$.

3.6.3. Associations between Insulin Levels and MCI. Five sets of data were extracted from 5 studies involving a total of 4653 individuals. A random-effects meta-analysis was performed, and the results showed that there were no significant differences in blood insulin levels between the MCI and HC groups (Hedges' $g=1.557,95 \% \mathrm{CI}=-0.253$ to 3.367 , and $P=0.092$; Figure 7 ). A sensitivity analysis was not performed because the number of studies was too small. However, we identified significant heterogeneity among the studies $\left(Q=264.128, I^{2}=98.486\right.$, and $\left.P<0.001\right)$.

3.6.4. Associations between Insulin Levels and Patients with Mild (or Light) Nonpsychotic Dementia. Eight sets of data were extracted from 8 studies involving a total of 543 individuals. A random-effects meta-analysis was performed, and the results showed that mild nonpsychotic dementia patients had significantly higher insulin levels than were observed in the HCs (Hedges' $g=0.520$, $95 \% \mathrm{CI}=0.284$ to 0.757 , and $P=0.000$; Figure 7 ). A sensitivity analysis indicated that these results were not unduly influenced by any particular study (Additional File 6). Furthermore, low heterogeneity was observed among the studies in this meta-analysis $\left(Q=11.531\right.$, d.f. $=7, I^{2}=$ 39.293, and $P=0.117$ ), demonstrating that the differences among the groups made a negligible contribution to this heterogeneity.

3.6.5. Associations between Insulin Levels and Patients with Moderate or Heavy Nonpsychotic Dementia. Twelve sets of data were extracted from 8 studies involving a total of 610 individuals. A random-effects meta-analysis was performed, and the results showed that the middle and heavy 
Meta-analysis

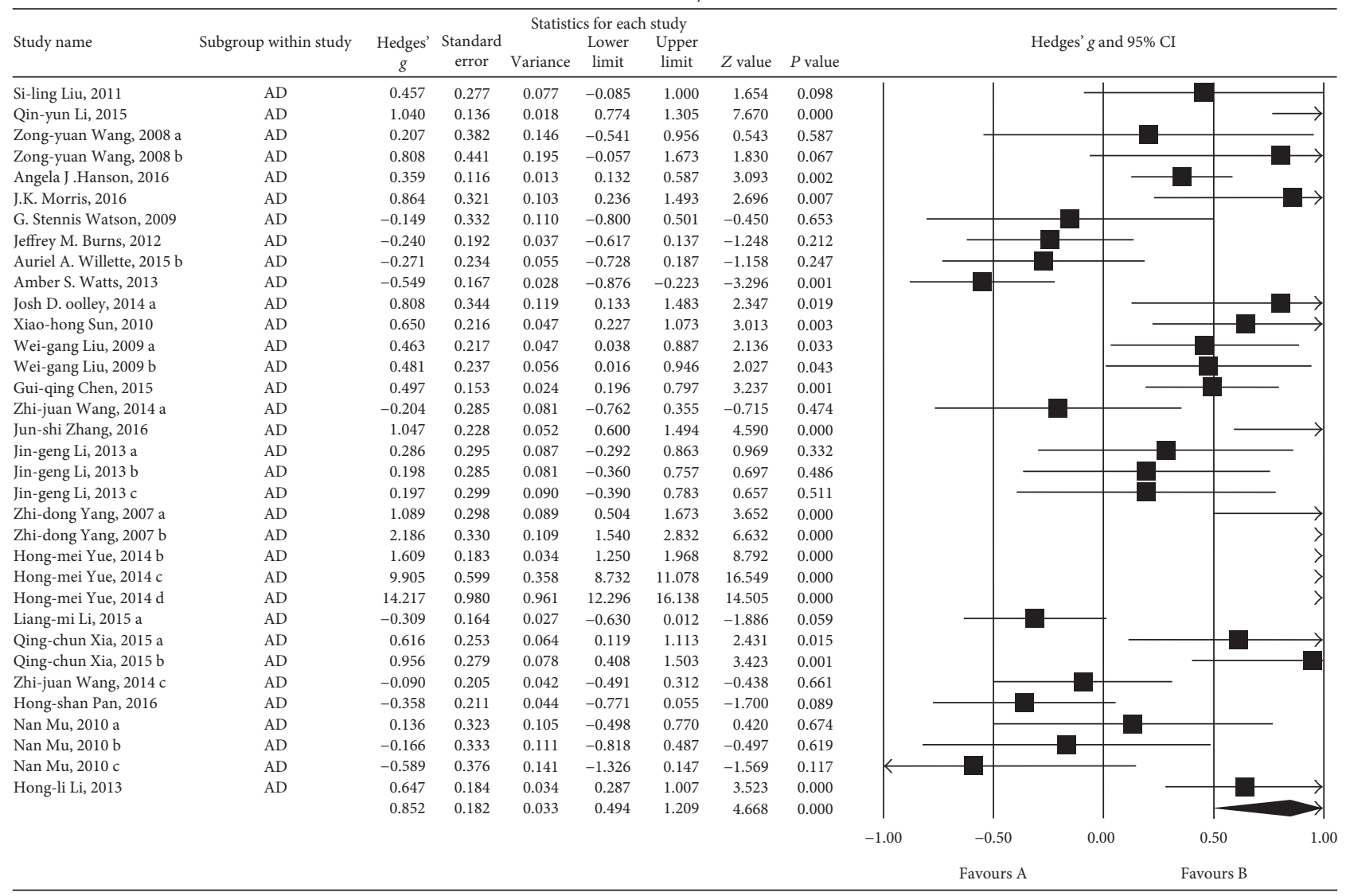

Figure 6: Forest plot of the random-effects meta-analysis of differences in blood insulin concentrations between AD patients and HC subjects. In all, 34 sets of data encompassing a total of 8407 individuals were included. The sizes of the squares are proportional to study weight. CI, confidence interval.

nonpsychotic dementia patients had significantly higher insulin levels than were observed in the HCs (Hedges' $g=2.379,95 \% \mathrm{CI}=1.007$ to 3.752 , and $P=0.001$; Figure 7 ). A sensitivity analysis indicated that the results were not unduly influenced by any particular study (Additional File 7). However, significant heterogeneity was observed among the studies in this meta-analysis $(Q=531.782$, d.f. $=11$, $I^{2}=97.931$, and $\left.P=0.000\right)$.

The results of the three subgroup analyses demonstrated that the high heterogeneity observed for increased insulin levels in the peripheral blood in patients with nonpsychotic dementia may have been caused by the severity of the dementia as follows: the greater the severity of nonpsychotic dementia, the higher the ESs of peripheral blood insulin levels. Compared with those of the HC group, the insulin levels in the MCI group were not correlated with the MCI.

In a series of metaregression analyses, we assessed whether gender or continuous variables, such as age, could explain the observed between-study differences. Gender was found to have a moderating effect $(P=0.29)$, but age did not significantly contribute to heterogeneity $(P<0.05)$.

Overall, our results indicated that the significant heterogeneity observed in the patients with increased insulin and nonpsychotic dementia could have been caused by a variety of factors, such as gender, sampling differences, and the severity of nonpsychotic dementia.

3.7. Publication Bias. In the abnormal insulin group, no significant publication bias was detected among the studies in a visual inspection of funnel plots (Additional File 8). These results were confirmed in Egger's tests $(t=0.007$, d.f. $=15$, and $P=0.497)$. Moreover, the nonpsychotic dementia group and $\mathrm{AD}$ group showed slight publication biases and had the following Egger's test values: for insulin, $t=3.26$, d.f. $=50$, and $P=0.001$ (Additional File 9); and for $\mathrm{AD}, t=2.399$, d.f. $=32$, and $P=0.011$ (Additional File 10). Because the ESs of small sample studies estimate a large amount of variation, the appearance of extreme ES values is more likely to emerge in small sample studies than in large sample studies. This publication bias was not statistically significant, and we therefore applied Duval and Tweedie's trim and fill method in further analyses.

The classic fail-safe $N$ was used to assess publication bias, and the results revealed that 4805 missing studies would be required for insulin and 1809 missing studies would be required for $\mathrm{AD}$ to achieve $P$ values of $>0.05$. These results support the notion that the observed publication bias was unlikely to have caused the positive results of our meta-analysis. 


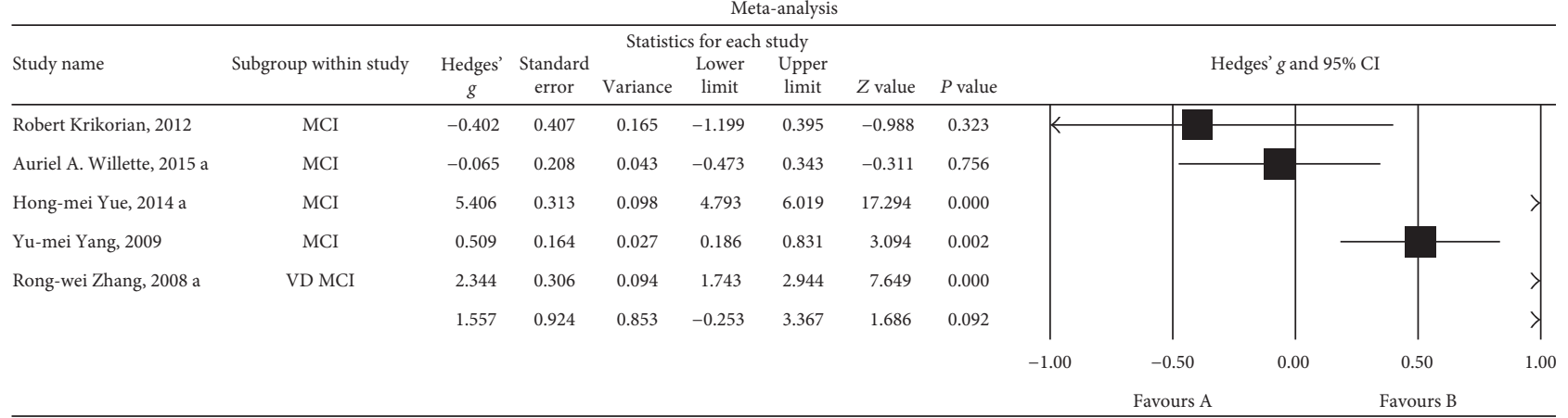

(a)

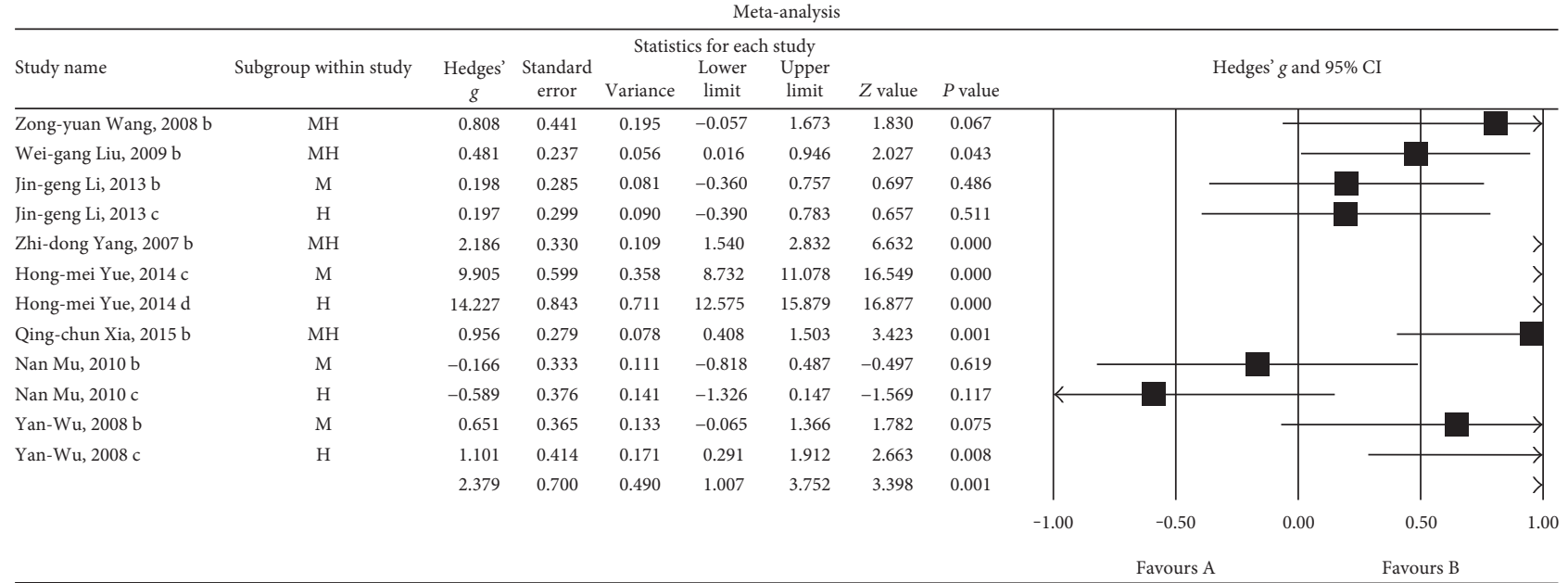

(b)

Meta-analysis

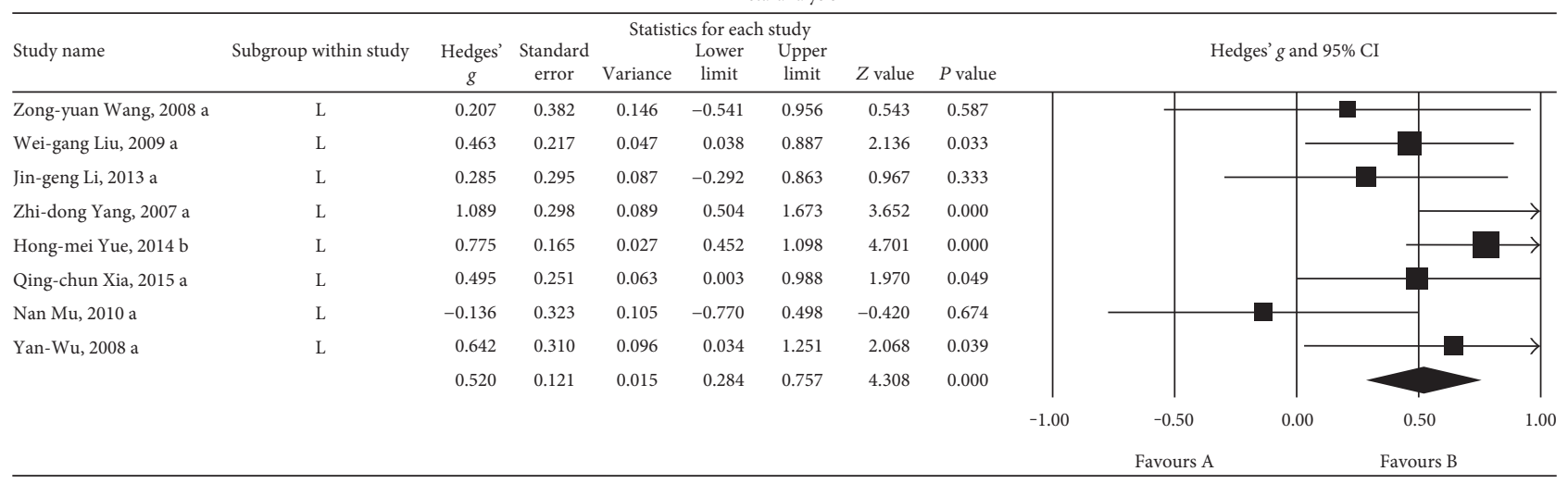

(c)

Figure 7: Forest plot of the random-effects meta-analysis of differences in blood insulin concentrations between patients with different severities of dementia and HC subjects. These 3 pictures in (a), (b), and (c) represent a comparison of insulin levels between HC subjects and MCI, mild (L), and moderate to severe $(\mathrm{MH})$ dementia patients, respectively.

\section{Discussion}

Many studies have focused on the changes that occur in the Pi3k-Akt insulin signaling pathway in nonpsychotic dementia patients. The Pi3k-Akt insulin signaling pathway is an important pathway in insulin metabolism and is also involved in growth and development, metabolism, and vital cognitive activities. Therefore, it is important to determine whether insulin plays a specific role in nonpsychotic dementia. Whether circulating levels of insulin are altered in nonpsychotic dementia patients has been controversial for a long time. Hence, the significance of insulin levels to the etiology of nonpsychotic dementia etiology was not fully known. The results of this study provide strong clinical evidence supporting the notion that nonpsychotic dementia is associated with increased levels of circulating 
insulin and decreased levels of insulin in the CSF. These results provide new insights into a potential molecular pathway that confers vulnerability to the development of nonpsychotic dementia. A strength of this study is that it is based on a large amount of data and a sufficient number of studies related to nonpsychotic dementia.

We explored the relationship between insulin levels and the occurrence of nonpsychotic dementia from three different perspectives. Our results show that CSF insulin levels are lower in patients with nonpsychotic dementia than in HCs, while insulin levels in the peripheral blood may be higher. Additionally, high insulin levels in the peripheral blood were associated with lower scores in cognitive functions. Because the number of studies that included a CSF group, a VD group, an MCI patient group, an L group, and an $\mathrm{MH}$ group was small, our results only reveal that there is a correlation between insulin levels and nonpsychotic dementia in these groups. The results suggest that insulin levels are higher in the peripheral blood in $\mathrm{VD}, \mathrm{AD}, \mathrm{L}$, and $\mathrm{MH}$ patients while CSF insulin levels are lower in nonpsychotic dementia patients. The ES was higher in the VD group than in the $\mathrm{AD}$ group and higher in the $\mathrm{MH}$ group than in the L group.

Our results show that there was no difference in insulin levels between MCI patients and HCs. Eight sets of data obtained from 4 studies support the finding that there is a relationship between CSF insulin levels and the severity of nonpsychotic dementia. Because the number of enrolled patients was small, the present results show that CSF insulin levels are lower in patients with severe nonpsychotic dementia, and there were no significant differences between MCI patients and HCs. Moreover, when the effects of drugs on insulin metabolism were excluded, a subgroup analysis showed that there was no correlation between nonpsychotic dementia and insulin levels. Therefore, the following two possibilities are considered. First, because MMSE scores were significantly lower in the high insulin subjects than in the HC group, the fact that there was no correlation may be related to the small sample size. Hence, as the study size increased, the trend toward an increase in serum insulin levels in the MCI patients might reach significance, and insulin levels are more likely to vary with the severity of the nonpsychotic dementia. Second, because the severity of nonpsychotic dementia increased with the ages of the affected patients, insulin levels may simply increase with age and may not be a key factor in the pathology of nonpsychotic dementia.

Several studies [44-46] have described insulin levels and MMSE scores in nonpsychotic dementia patients without diabetes, type 2 diabetes patients without dementia, and patients with nonpsychotic dementia and type 2 diabetes, and these studies have reported that there are no significant differences in the ages or genders of these patients. Insulin levels increased in the nonpsychotic dementia patients, and cognitive function scores decreased in subjects with high insulin levels. Further investigations of insulin levels in MCI patients are required to confirm these results.

In this meta-analysis, we found various levels of betweenstudy heterogeneity in the outcomes. Although we attempted to adjust for potential confounders, none of the theoretically relevant categorical or continuous variables that were tested explained the observed heterogeneity. A subgroup analysis showed that serum insulin levels (as opposed to plasma levels) were significantly higher in nonpsychotic dementia patients than in HC subjects and that VD insulin levels (as opposed to AD levels) were significantly higher in nonpsychotic dementia patients than in HC subjects. However, the high level of between-study heterogeneity was not lower in these subgroup analyses. MH insulin levels (but not L levels) were significantly higher in nonpsychotic dementia patients than in HC subjects, and low heterogeneity was found in the $\mathrm{L}$ group analysis. The high level of between-study heterogeneity was not reduced in the $\mathrm{MH}$ and drug intervention elimination groups. This high level of between-study heterogeneity may have been caused by the differences in the samples (i.e., according to gender, age, or sample type), the type of nonpsychotic dementia, the degree of nonpsychotic dementia, or the drug intervention. In addition, hunger, cold, exogenous diet stimulation, endocrine diseases, and the selection of inspection methods and reagents could potentially have caused an abnormal secretion of insulin. Hence, potential confounders that could contribute to the observed between-study heterogeneity were present in a variety of the analyzed studies and may have been related to patient conditions during sample extraction. Because insulin levels that are determined using blood samples can be affected by handling time and the state of the research subjects, studies that include patients who provide samples while on an empty stomach and who are not affected by other endocrine factors may be required to further investigate whether these factors contributed to the variance observed among the studies in this analysis.

\section{Conclusions}

Overall, CSF insulin levels were lower in nonpsychotic dementia patients (enrolled sample number $<10$, more experimental data are needed to support this finding). The high insulin subjects had significantly lower MMSEs and nonpsychotic dementia events than were observed in the HCs, and blood insulin levels were significantly higher in nonpsychotic dementia patients than in HCs. These results indicate that insulin levels are an important indicator that may be useful in clinical diagnoses of nonpsychotic dementia.
Abbreviations
A $342: \quad$ Beta-amyloid 42
AD: Alzheimer's disease
APP: Amyloid precursor protein
CSF: Cerebrospinal fluid
ESs: $\quad$ Effect sizes
HC: Healthy control
HCs: Healthy control subjects
L: $\quad$ Light/mild
MH: $\quad$ Middle and heavy
MCI: Mild cognitive impairment
MMSE: Mini-Mental State Examination
NOS: Newcastle-Ottawa Scale 
PRISMA: Reporting Items for Systematic Reviews and Meta-Analysis

s.d.: $\quad$ Standard deviation

VD: $\quad$ Vascular dementia

95\% CI: $95 \%$ confidence interval.

\section{Additional Points}

Availability of Data and Material. The datasets supporting the conclusions of this article are included within the article and its additional files.

\section{Disclosure}

The funders had no role in the study design, analysis, decision to publish, or preparation of the manuscript.

\section{Conflicts of Interest}

The authors declare that there is no conflict of interest regarding the publication of this article.

\section{Authors' Contributions}

Jia-xu Chen and Qiu-xia Pan raised the idea of this study. Qiu-xia Pan and Xiao-juan Li contributed equally as first authors of this manuscript and were the two main reviewers of this study. Fang-fang Wang and Ya-jing Hou contributed to the data collection. You-ming Jiang provided guidance regarding the methodology. Yue-yun Liu took part in the data selection and extraction. Zhi-yi Yan participated in the data extraction. Qiu-xia Pan, Qing-lai Bian, and Wen-qi Qiu cocompleted the primary draft. All authors contributed to the design of this meta-analysis and played a substantial role in the manuscript redaction. All authors approved the final version of this article.

\section{Acknowledgments}

The authors thank Yao-wen Zhang for the very helpful and constructive suggestions related to data extraction during this study. This work was funded by the National Natural Science Foundation of China (Grant nos. 81473597 and 81630104), the Beijing Natural Sciences Foundation (Grant no. 7152093 ), and the 111 Project (no. B07007).

\section{Supplementary Materials}

Supplementary 1. Sensitivity analysis of differences in insulin levels in the CSF between nonpsychotic dementia patients and $\mathrm{HC}$ subjects.

Supplementary 2. Sensitivity analysis of the random-effects meta-analysis of differences in MMSE scores between patients with abnormal insulin levels and HC subjects.

Supplementary 3. Sensitivity analysis of differences in blood insulin levels between nonpsychotic dementia patients and HC subjects.
Supplementary 4. Sensitivity analysis of differences in blood insulin levels between vascular dementia (VD) patients and HC subjects.

Supplementary 5. Sensitivity analysis of differences in blood insulin levels between $\mathrm{AD}$ patients and $\mathrm{HC}$ subjects.

Supplementary 6. Sensitivity analysis of differences in insulin levels between L (light/mild) nonpsychotic dementia patients and $\mathrm{HC}$ subjects.

Supplementary 7. Sensitivity analysis of differences in insulin levels between $\mathrm{MH}$ (moderate to heavy) nonpsychotic dementia patients and $\mathrm{HC}$ subjects.

Supplementary 8. Publication bias in MMSE scores between patients with abnormal insulin levels and HC subjects.

Supplementary 9. Publication bias in insulin levels between nonpsychotic dementia patients and HC subjects.

Supplementary 10. Publication bias in insulin levels between $\mathrm{AD}$ patients and $\mathrm{HC}$ subjects.

Supplementary 11. PRISMA 2009 checklist.

Supplementary 12. PRISMA 2009 flow diagram.

\section{References}

[1] C. U. Onyike, "Psychiatric aspects of dementia," CONTINUUM: Lifelong Learning in Neurology, vol. 22, no. 2, pp. 600-614, 2016.

[2] H. Umegaki, T. Kawamura, T. Umemura, and N. Kawano, "Factors associated with cognitive decline in older adults with type 2 diabetes mellitus during a 6-year observation," Geriatrics \& Gerontology International, vol. 15, no. 3, pp. 302-310, 2015.

[3] Z. Chen, Y. Y. Xu, R. Wu et al., "Impaired learning and memory in rats induced by a high-fat diet: involvement with the imbalance of nesfatin- 1 abundance and copine 6 expression," Journal of Neuroendocrinology, vol. 29, no. 4, 2017.

[4] A. L. Kauffman, J. M. Ashraf, M. R. Corces-Zimmerman, J. N. Landis, and C. T. Murphy, "Insulin signaling and dietary restriction differentially influence the decline of learning and memory with age," PLoS Biology, vol. 8, no. 5, article e1000372, 2010.

[5] Q. C. Li, G. I. Wand, B. Y. Li, H. Sun, and N. C. Jiang, "The effect of insulin on the spatial learning and memory abilities of the Alzheimer's disease model rats," Zhongguo Ying Yong Sheng Li Xue Za Zhi, vol. 24, no. 4, pp. 494-498, 2008.

[6] J. Q. Wang, J. Yin, Y. F. Song et al., "Brain aging and AD-like pathology in streptozotocin-induced diabetic rats," Journal of Diabetes Research, vol. 2014, Article ID 796840, 12 pages, 2014.

[7] A. M. Dorrance, N. Matin, and P. W. Pires, "The effects of obesity on the cerebral vasculature," Current Vascular Pharmacology, vol. 12, no. 3, pp. 462-472, 2014.

[8] J. M. Burns, R. A. Honea, E. D. Vidoni, L. J. Hutfles, W. M. Brooks, and R. H. Swerdlow, "Insulin is differentially related to cognitive decline and atrophy in Alzheimer's disease and 
aging," Biochimica et Biophysica Acta (BBA) - Molecular Basis of Disease, vol. 1822, no. 3, pp. 333-339, 2012.

[9] A. M. Dorrance, N. Matin, and P. W. Pires, "The effects of obesity on the cerebral vasculature," Current Vascular Pharmacology, vol. 12, no. 3, pp. 462-472, 2014.

[10] A. M. Dorrance, N. C. Rupp, and E. F. Nogueira, "Mineralocorticoid receptor activation causes cerebral vessel remodeling and exacerbates the damage caused by cerebral ischemia," Hypertension, vol. 47, no. 3, pp. 590-595, 2006.

[11] S. Craft, L. D. Baker, T. J. Montine et al., "Intranasal insulin therapy for Alzheimer disease and amnestic mild cognitive impairment: a pilot clinical trial," Archives of Neurology, vol. 69, no. 1, pp. 29-38, 2012.

[12] R. O. Roberts, Y. E. Geda, D. S. Knopman et al., "Association of duration and severity of diabetes mellitus with mild cognitive impairment," Archives of Neurology, vol. 65, no. 8, pp. 1066-1073, 2008.

[13] J. Y. Jeon, S. R. Kim, H. J. Kim et al., "Risk factors of severe hypoglycemia requiring medical assistance and neurological sequelae in patients with diabetes: a case-control study," Medicine, vol. 95, no. 47, article e5365, 2016.

[14] H. Kenna, F. Hoeft, R. Kelley et al., "Fasting plasma insulin and the default mode network in women at risk for Alzheimer's disease," Neurobiology of Aging, vol. 34, no. 3, pp. 641-649, 2013.

[15] M. Mwamburi and W. Q. Qiu, "Different associations of premorbid intelligence $v s$. current cognition with BMI, insulin and diabetes in the homebound elderly," Integrative Molecular Medicine, vol. 3, no. 3, pp. 547-552, 2016.

[16] A. Liberati, D. G. Altman, J. Tetzlaff et al., "The PRISMA statement for reporting systematic reviews and metaanalyses of studies that evaluate health care interventions: explanation and elaboration," Journal of Clinical Epidemiology, vol. 62, no. 10, pp. e1-e34, 2009.

[17] R. L. Ownby, E. Crocco, A. Acevedo, V. John, and D. Loewenstein, "Depression and risk for Alzheimer disease: systematic review, meta-analysis, and metaregression analysis," Archives of General Psychiatry, vol. 63, no. 5, pp. 530538, 2006.

[18] L. Chen, The Level of Risk Factors for Cognitive Impairment in Insulin Resistance and Diabetes Rat Brain, [M.Sc. Thesis], Hebei Medical University, 2009.

[19] Q. H. Fang, Risk Factors of Mild Cognitive Impairment in Type 2 Diabetes, [M.Sc. Thesis], Anhui Medical University, 2014.

[20] D. H. Liu, Resting-State Functional MRI Study on the Cognitive Impairment in Type 2 Diabetes Mellitus, [M.Sc. Thesis], Third Military Medical University, 2015.

[21] J. K. Liu, The Research of mTOR and Tau Phosphorylated Protein in Hippocampus Tissue of Type 2 Diabetes and Alzheimer's Disease Rats, [M.Sc. Thesis], Nanchang University, 2012.

[22] Y. F. Mao, The Function and Mechanism of Intranasal Insulin on Alzheimer's Disease, Dr. Dissertation, Zhejiang University, 2016.

[23] W. Shen, Resting-State Functional Magnetic Resonance Imaging and Neurocognitive Function in Type 2 Diabetes Mellitus, [M.Sc. Thesis], Southern Medical University, 2014.

[24] Y. Wang, Relationship between Cognitive Dysfunction and Vascular Complications in Patients with Type 2 Diabetes Mellitus, [M.Sc. Thesis], Shandong University, 2015.
[25] H. Xiong, L. Ding, W. JJ et al., "Effect the impact of insulin on learning and memory of Alzheimer-like mice and its mechanism," Tianjin Medical Journal, vol. 41, no. 04, pp. 337-340, 2013.

[26] Y. Y. Xiong, Prevalence and Risk Factors for Dementia in Diabetics: A Community-Based Case-Control Study [M.Sc. Thesis], Fudan University, 2008.

[27] H. Y. Yu, Q. S. Wang, P. Li, and B. X. Yang, "The placebocontrolled study of type 2 diabetes patients with mild cognitive impairment," Chinese Journal of Gerontology, vol. 21, pp. 4087-4089, 2011.

[28] T. R. Bomfim, L. Forny-Germano, L. B. Sathler et al., "An anti-diabetes agent protects the mouse brain from defective insulin signaling caused by Alzheimer's disease-associated A $\beta$ oligomers," The Journal of Clinical Investigation, vol. 122, no. 4, pp. 1339-1353, 2012.

[29] D. A. Costello, M. Claret, H. Al-Qassab et al., "Brain deletion of insulin receptor substrate 2 disrupts hippocampal synaptic plasticity and metaplasticity," PLoS One, vol. 7, no. 2, article e31124, 2012.

[30] M. Hokama, S. Oka, J. Leon et al., "Altered expression of diabetes-related genes in Alzheimer's disease brains: the Hisayama study," Cerebral Cortex, vol. 24, no. 9, pp. 2476-2488, 2014.

[31] S. M. Hoscheidt, E. J. Starks, J. M. Oh et al., "Insulin resistance is associated with increased levels of cerebrospinal fluid biomarkers of Alzheimer's disease and reduced memory function in at-risk healthy middle-aged adults," Journal of Alzheimer's Disease, vol. 52, no. 4, pp. 1373-1383, 2016.

[32] S. Maimaiti, K. L. Anderson, C. DeMoll et al., "Intranasal insulin improves age-related cognitive deficits and reverses electrophysiological correlates of brain aging," The Journals of Gerontology Series A: Biological Sciences and Medical Sciences, vol. 71, no. 1, pp. 30-39, 2016.

[33] T. M. van Himbergen, A. S. Beiser, M. Ai et al., "Biomarkers for insulin resistance and inflammation and the risk for allcause dementia and Alzheimer disease: results from the Framingham Heart Study," Archives of Neurology, vol. 69, no. 5, pp. 594-600, 2012.

[34] W. L. Xu, N. L. Pedersen, L. Keller et al., "HHEX_23 AA genotype exacerbates effect of diabetes on dementia and Alzheimer disease: a population-based longitudinal study," PLoS Medicine, vol. 12, no. 7, article e1001853, 2015.

[35] X. J. Han, P. Yan, L. Wang, L. Cong, and Y. F. Du, "A preliminary study on the changes of insulin signaling pathway in Alzheimer disease," in National Symposium on Senile Dementia and Related Diseases, vol. 2013, pp. 74-75, Chinese Pharmacological Society, Nanjing, China, 2013.

[36] Y. Z. Rong, Y. J. Xie, X. M. Chen, and B. R. Zhou, “Analysis of the correlation among insulin resistance ApoE gene polymorphism and mild cognitive impairment," Hebei Medicine, vol. 19, no. 11, pp. 1604-1607, 2013.

[37] A. Ernst, A. N. Sharma, K. M. Elased, P. C. Guest, H. Rahmoune, and S. Bahn, "Diabetic $d b / d b$ mice exhibit central nervous system and peripheral molecular alterations as seen in neurological disorders," Translational Psychiatry, vol. 3, article e263, 2013.

[38] A. Jayaraman, D. Lent-Schochet, and C. J. Pike, "Diet-induced obesity and low testosterone increase neuroinflammation and impair neural function," Journal of Neuroinflammation, vol. 11, p. 162, 2014. 
[39] S. D. Ostergaard, S. Mukherjee, S. J. Sharp et al., “Associations between potentially modifiable risk factors and Alzheimer disease: a Mendelian randomization study," PLoS Medicine, vol. 12, no. 6, article e1001841, 2015.

[40] J. J. He, Y. Z. Zhang, and X. F. Zhang, "The relationship of cognitive dysfunction in elderly diabetic patients with insulin resistance and care analysis," China Modern Doctor, vol. 52, no. 28, pp. 138-140, 2014.

[41] Q. C. Li, G. L. Wang, B. Y. Li, H. Sun, and N. C. Jiang, "Effects of insulin on spatial learning and memory in AD rats," Chinese Journal of Applied Physiology, vol. 24, no. 04, pp. 494-498, 2008.

[42] Y. Yang, D. L. Ma, Y. P. Wang, T. Jiang, S. H. Hu, and X. F. $\mathrm{Yu}$, "Intranasal insulin treatment ameliorates Alzheimer disease-like changes in hippocampus in a rat model of type 2 diabetes," Chinese Journal of Pathophysiology, vol. 29, no. 01, pp. 56-61, 2013.

[43] T. Kawamura, T. Umemura, and N. Hotta, "Cognitive impairment in diabetic patients: can diabetic control prevent cognitive decline?," Journal of Diabetes Investigation, vol. 3, no. 5, pp. 413-423, 2012.

[44] L. J. Cao, "Relationship between cognitive dysfunction and insulin resistance in elderly patients with diabetes mellitus and nursing care," Nurses Training, vol. 28, no. 03, pp. 266267, 2013.

[45] J. Dong, "Relationship between cognitive dysfunction and insulin resistance in elderly patients with diabetes mellitus and nursing care," World Latest Medicine Information, vol. 15, no. 105, pp. 303-306, 2015.

[46] M. Li, L. Ma, Y. Fan, Z. Q. Song, and J. Li, "Effect of insulin on patients with type 2 diabetes mellitus complicated with mild cognitive impairment," Cardiovascular Disease Journal of of integrated Traditional Chinese and Western Medicine, vol. 14, no. 20, pp. 2417-20, 2016.

[47] N. Mu, S. C. Xu, Q. Chang, D. P. Rao, J. P. Chen, and C. Ma, "Serum lipids, insulin metabolism and 311 gene polymorphism of paraoxonase-2 in patients with different subtypes of Alzheimer's disease," Chinese Journal of Gerontology, vol. 34, pp. 5346-5348, 2014.

[48] Z. Shen and Y. Lin, "Significance of fasting serum insulin and C peptide in patients with Alzheimer's disease," Chinese Journal of Clinical Healthcare, vol. 15, no. 01, pp. 61-62, 2012.

[49] L. Su, Q. W. Zhang, W. Li et al., "Effect of course on cognitive function and related risk factors in elderly patients with type 2 diabetes mellitus," Chinese Journal of Geriatric Heart BrainVessel Diseases, vol. 17, no. 10, pp. 1071-1075, 2015.

[50] L. Y. Tang and W. Tang, "Effect of blood glucose fluctuation on the treatment of senile dementia," The Word Clinical Medicine, vol. 9, no. 7, pp. 67-68, 2015.

[51] Q. Tao, "The risk factors of senile patients with type II diabetes," Modern Hospital, vol. 15, no. 03, pp. 152-153, 2015.

[52] Q. H. Zhang and Y.F. Du, "Study on the relationship between cognitive impairment and serum levels of TNF- $\alpha$ and IL- 6 in middle-aged and elderly patients with type 2 diabetes mellitus," in National Symposium on Senile Dementia and Related Diseases, vol. 2013, pp. 67-68, Nanjing: Chinese Pharmacological Society, Nanjing, China, 2013.

[53] M. W. Akhtar, S. Sanz-Blasco, N. Dolatabadi et al., "Elevated glucose and oligomeric $\beta$-amyloid disrupt synapses via a common pathway of aberrant protein S-nitrosylation," Nature Communications, vol. 7, p. 10242, 2016.

[54] A. M. Barron, E. R. Rosario, R. Elteriefi, and C. J. Pike, "Sex-specific effects of high fat diet on indices of metabolic syndrome in 3xTg-AD mice: implications for Alzheimer's disease," PLoS One, vol. 8, no. 10, article e78554, 2013.

[55] L. Devi, M. J. Alldred, S. D. Ginsberg, and M. Ohno, "Mechanisms underlying insulin deficiency-induced acceleration of $\beta$-amyloidosis in a mouse model of Alzheimer's disease," PLoS One, vol. 7, no. 3, article e32792, 2012.

[56] Y. D. Ke, F. Delerue, A. Gladbach, J. Gotz, and L. M. Ittner, "Experimental diabetes mellitus exacerbates tau pathology in a transgenic mouse model of Alzheimer's disease," PLoS One, vol. 4, no. 11, article e7917, 2009.

[57] J. J. Ramos-Rodriguez, S. Molina-Gil, O. Ortiz-Barajas et al., "Central proliferation and neurogenesis is impaired in type 2 diabetes and prediabetes animal models," PLoS One, vol. 9, no. 2, article e89229, 2014.

[58] P. Serbedzija, J. E. Madl, and D. N. Ishii, "Insulin and IGF-I prevent brain atrophy and DNA loss in diabetes," Brain Research, vol. 1303, pp. 179-194, 2009.

[59] K. Talbot, H. Y. Wang, H. Kazi et al., "Demonstrated brain insulin resistance in Alzheimer's disease patients is associated with IGF-1 resistance, IRS-1 dysregulation, and cognitive decline," The Journal of Clinical Investigation, vol. 122, no. 4, pp. 1316-1338, 2012.

[60] H. Y. Mu, "Comparative analysis of insulin resistance in patients with Alzheimer's disease and type 2 diabetes mellitus," in The 4th National Symposium on Dementia and Cognitive Impairment and Senior Speeches, vol. 2015, p. 173, Beijing: Chinese Medical Association, Beijing, China, 2015.

[61] C. G. Jolivalt, R. Hurford, C. A. Lee, W. Dumaop, E. Rockenstein, and E. Masliah, "Type 1 diabetes exaggerates features of Alzheimer's disease in APP transgenic mice," Experimental Neurology, vol. 223, no. 2, article 19931251, pp. 422-431, 2010.

[62] Y. F. Mao, Z. Guo, T. Zheng et al., "Intranasal insulin alleviates cognitive deficits and amyloid pathology in young adult APPswe/PS1dE9 mice," Aging Cell, vol. 15, no. 5, pp. 893-902, 2016.

[63] M. Solas, B. Aisa, M. C. Mugueta, R. J. Del, R. M. Tordera, and M. J. Ramirez, "Interactions between age, stress and insulin on cognition: implications for Alzheimer's disease," Neuropsychopharmacology, vol. 35, no. 8, pp. 1664-1673, 2010.

[64] M. Chen, P. Y. Shan, A. F. Liu, L. Ma, and X. L. Yu, "Plasma fasting glucose, fasting insulin and insulin degrading enzyme in Alzheimer's disease," Journal of Shandong University (Health Sciences), vol. 50, no. 03, pp. 75-78, 2012.

[65] R. Y. Huang, J. J. Ren, H. Y. Han, and M. N. Tang, "The correlation between metabolic syndrome and Alzheimer's disease research," Journal of Psychiatry, vol. 26, no. 03, pp. 175-177, 2013.

[66] G. X. Wang, "Correlation analysis of insulin resistance and cognitive impairment," China Practical Medicine, vol. 6, no. 26, pp. 38-39, 2011.

[67] H. M. Kim, J. M. Seong, and J. Kim, "Risk of hospitalization for hypoglycemia among older Korean people with diabetes mellitus: interactions between treatment modalities and comorbidities," Medicine, vol. 95, no. 42, article e5016, 2016.

[68] N. L. Rasgon, H. A. Kenna, T. E. Wroolie et al., "Insulin resistance and hippocampal volume in women at risk for 
Alzheimer's disease," Neurobiology of Aging, vol. 32, no. 11, pp. 1942-1948, 2011.

[69] E. M. Schrijvers, J. C. Witteman, E. J. Sijbrands, A. Hofman, P. J. Koudstaal, and M. M. Breteler, "Insulin metabolism and the risk of Alzheimer disease: the Rotterdam study," Neurology, vol. 75, no. 22, pp. 1982-1987, 2010.

[70] Z. S. Tan, A. S. Beiser, C. S. Fox et al., "Association of metabolic dysregulation with volumetric brain magnetic resonance imaging and cognitive markers of subclinical brain aging in middle-aged adults: the Framingham Offspring Study," Diabetes Care, vol. 34, no. 8, pp. 1766-1770, 2011.

[71] M. K. Townsend, O. I. Okereke, W. Xia, T. Yang, D. J. Selkoe, and F. Grodstein, "Relation between insulin, insulin-related factors, and plasma amyloid beta peptide levels at midlife in a population-based study," Alzheimer Disease and Associated Disorders, vol. 26, no. 1, pp. 50-54, 2012.

[72] M. van Oijen, O. I. Okereke, J. H. Kang et al., "Fasting insulin levels and cognitive decline in older women without diabetes," Neuroepidemiology, vol. 30, no. 3, pp. 174-179, 2008.

[73] A. A. Willette, S. C. Johnson, A. C. Birdsill et al., "Insulin resistance predicts brain amyloid deposition in late middleaged adults," Alzheimers Dement, vol. 11, no. 5, pp. 504510, 2015.

[74] T. T. Huang, "Effects of insulin resistance on cognitive impairment in patients with Parkinson's disease," Journal of Bethune Medical Science, vol. 13, no. 02, pp. 129-130, 2015.

[75] M. Chen, Relationship of Metabolism Related Factors and Alzheimer's Disease [M.Sc. Thesis], Shandong University, 2012.

[76] Z. S. Deng, Activity of Cholinesterase and Expression of ChAT, Aßof Brain Tissue in Type-2 Diabetic Rat [M.Sc. Thesis], Shanxi Medical University, 2008.

[77] W. G. Liu, R. H. Liu, L. Li et al., "Study of CSF levels of glucose, true insulin, $\mathrm{A} \beta_{40}$ and $\mathrm{A} \beta_{42}$ in patients with Alzheimer's disease," Journal of Brain and Nervous Diseases, vol. 17, no. 06, pp. 434-436, 2009.

[78] W. G. Liu, R. Z. Tian, Y. Q. Feng et al., "Level of fasting plasma glucose, true insulin, C-peptide and amyloid Betaprotein in patients with Alzheimer disease," Chinese Rehabilitation Theory and Practice, vol. 13, no. 07, pp. 645-646, 2007.

[79] Y. Q. Ma, Study on the Relationship between PI3K/PKB/ mTOR/S6K1 Signaling Pathway and Type 2 Diabetes Mellitus and Alzheimer's Disease, Dr. Dissertation, Nanchang University, 2013.

[80] L. Zhang, Y. Fang, Y. J. Lian, J. K. Wang, Y. K. Zheng, and Z. Y. Luo, "Study on relationship between the insulin resistant with the concentration of tau protein and $\beta$-amyloid protein in cerebrospinal fluid of patients with mild cognitive impairment," Modern Preventive Medicine, vol. 38, no. 09, pp. 1774-1775, 2011.

[81] X. H. Zhao, Y. M. Yang, J. Bao, X. Wang, Y. Y. Zhang, and Q. Zhao, "Clinical study on relationship between metabolic syndrome and mild cognitive impairment," Journal of Kunming Medical University, vol. 05, pp. 82-86, 2012.

[82] E. Grunblatt, J. Bartl, D. I. Iuhos et al., "Characterization of cognitive deficits in spontaneously hypertensive rats, accompanied by brain insulin receptor dysfunction," Journal of Molecular Psychiatry, vol. 3, no. 1, p. 6, 2015.

[83] W. J. Katon, E. H. Lin, L. H. Williams et al., "Comorbid depression is associated with an increased risk of dementia diagnosis in patients with diabetes: a prospective cohort study," Journal of General Internal Medicine, vol. 25, no. 5, pp. 423-429, 2010.

[84] L. Ma, M. Feng, Y. Qian et al., "Insulin resistance is an important risk factor for cognitive impairment in elderly patients with primary hypertension," Yonsei Medical Journal, vol. 56, no. 1, pp. 89-94, 2015.

[85] M. D. Sullivan, W. J. Katon, L. C. Lovato et al., "Association of depression with accelerated cognitive decline among patients with type 2 diabetes in the ACCORD-MIND trial," JAMA Psychiatry, vol. 70, no. 10, pp. 1041-1047, 2013.

[86] B. Y. Ding, The Incidence and Related Risk Factors of Vascular Cognitive Impairment Not Dementia in Inpatients with Diabetes Mellitus Type 2 and Acute Cerebral Infarction, [M.Sc. Thesis], Dalian Medical University, 2015.

[87] J. Hou, The Study for the Change of CMBs-Related Memory Function and Resting-State Brain Function in T2DM with CMBs, [M.Sc. Thesis], Central South University, 2014.

[88] C. H. Shao, K. Li, and X. B. Cao, "Hypertension and related risk factors of vascular dementia," Chinese Focus Journal, vol. 27, no. 01, pp. 13-15, 2012.

[89] X. Zhang, The Control of Glycated Hemoglobin Level and the Use of Insulin Affect the Cognitive Function in Type2 Diabetes, [M.Sc. Thesis], Third Military Medical University, 2014.

[90] X. Liu, Y. N. Zhang, L. H. Jiang, and W. Li, “Abnormal expression of insulin receptor and insulin-like growth factor I receptor in hippocampus of Alzheimer's disease," Chinese Journal of Gerontology, vol. 07, pp. 1191-1194, 2011.

[91] Y. Ma, D. Wu, W. Zhang, J. Liu, S. Chen, and B. Hua, "Investigation of PI3K/PKB/mTOR/S6K1 signaling pathway in relationship of type 2 diabetes and Alzheimer's disease," International Journal of Clinical and Experimental Medicine, vol. 8, no. 10, pp. 18581-18590, 2015.

[92] Z. S. Deng, S. L. Zhang, Y. Zhang, Y. Chen, and X. J. Yang, "The study of expression of $\beta$-amyloid of serum and brain tissue in type-2 diabetic rat," Journal of Practical Medical Techniques, vol. 15, no. 12, pp. 1503-1505, 2008.

[93] X. Gao, D. Zhao, H. J. Cui, and F. Y. Li, "Study on expression of $\mathrm{A} \beta$ in serum and brain tissues in rats with type 2 diabetes mellitus and its relationship with Alzheimer's disease," Hebei Medical Journal, vol. 34, no. 22, pp. 3386-3387, 2012.

[94] A. H. Lv, S. K. Zhu, and X. M. Zhang, "Effect of insulin resistance on the expression of $\mathrm{A} \beta$ protein in hippocampus of rats and the change of cognitive behavior," Chinese Journal of Practical Nervous Diseases, vol. 14, no. 21, pp. 40-41, 2011.

[95] S. H. Yuan, S. Z. Gao, X. P. Liu et al., "The change of cognition function in insulin resistance rats and its brain tissues with Alzheimer-like disease," Stroke and Neurological Disorders, vol. 17, no. 03, pp. 139-143, 2010.

[96] X. J. Zhang, S. S. Yang, M. X. Zhang, and Y. Yang, "Common pathogenesis of type 2 diabetes and Alzheimer's disease: a potential mechanism," Chinese Journal of Pathophysiology, vol. 26, no. 06, pp. 1107-1114, 2010.

[97] D. Baumeister, R. Akhtar, S. Ciufolini, C. M. Pariante, and V. Mondelli, "Childhood trauma and adulthood inflammation: a meta-analysis of peripheral C-reactive protein, interleukin- 6 and tumour necrosis factor- $\alpha$," Molecular Psychiatry, vol. 21, no. 5, pp. 642-649, 2016.

[98] J. Bowden, J. F. Tierney, A. J. Copas, and S. Burdett, "Quantifying, displaying and accounting for heterogeneity in the 
meta-analysis of RCTs using standard and generalised Q statistics," BMC Medical Research Methodology, vol. 11, p. 41, 2011.

[99] A. Masi, D. S. Quintana, N. Glozier, A. R. Lloyd, I. B. Hickie, and A. J. Guastella, "Cytokine aberrations in autism spectrum disorder: a systematic review and meta-analysis," Molecular Psychiatry, vol. 20, no. 4, pp. 440-446, 2015.

[100] P. Langhorne, "Bias in meta-analysis detected by a simple, graphical test. Bias in meta-analysis detected by a simple, graphical test. Prospectively identified trials could be used for comparison with meta-analyses," BMJ, vol. 316, no. 7129, article 9492689, p. 471, 1998.

[101] K. L. Soeken and A. Sripusanapan, "Assessing publication bias in meta-analysis," Nursing Research, vol. 52, no. 1, pp. 57-60, 2003. 

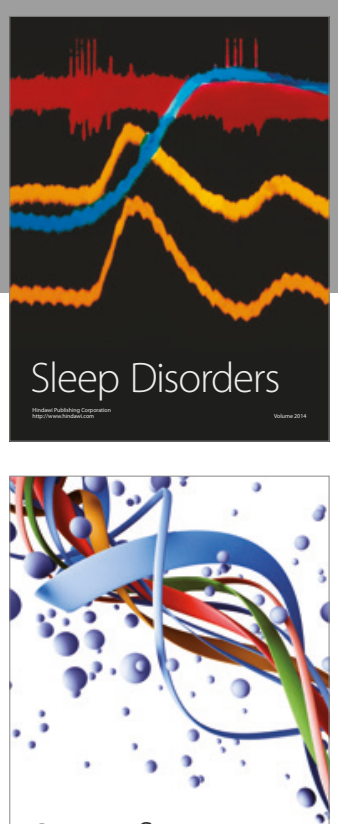

Scientifica
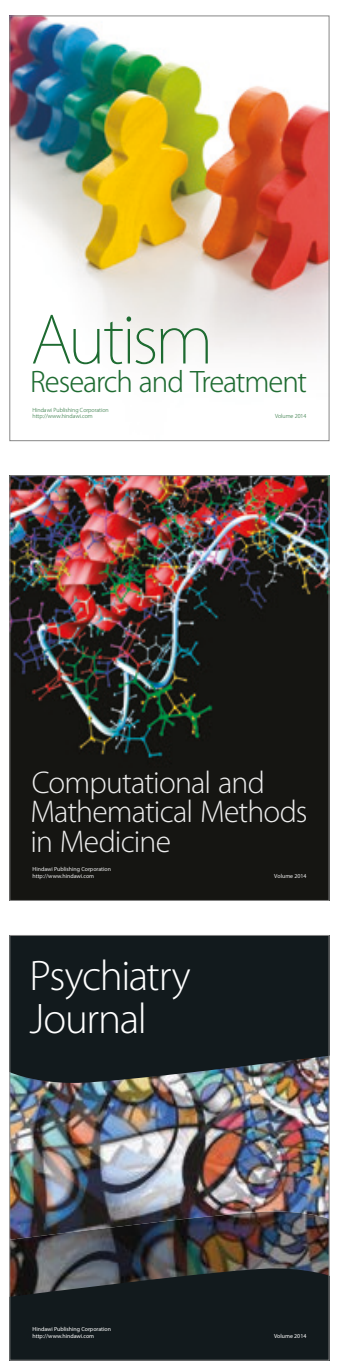
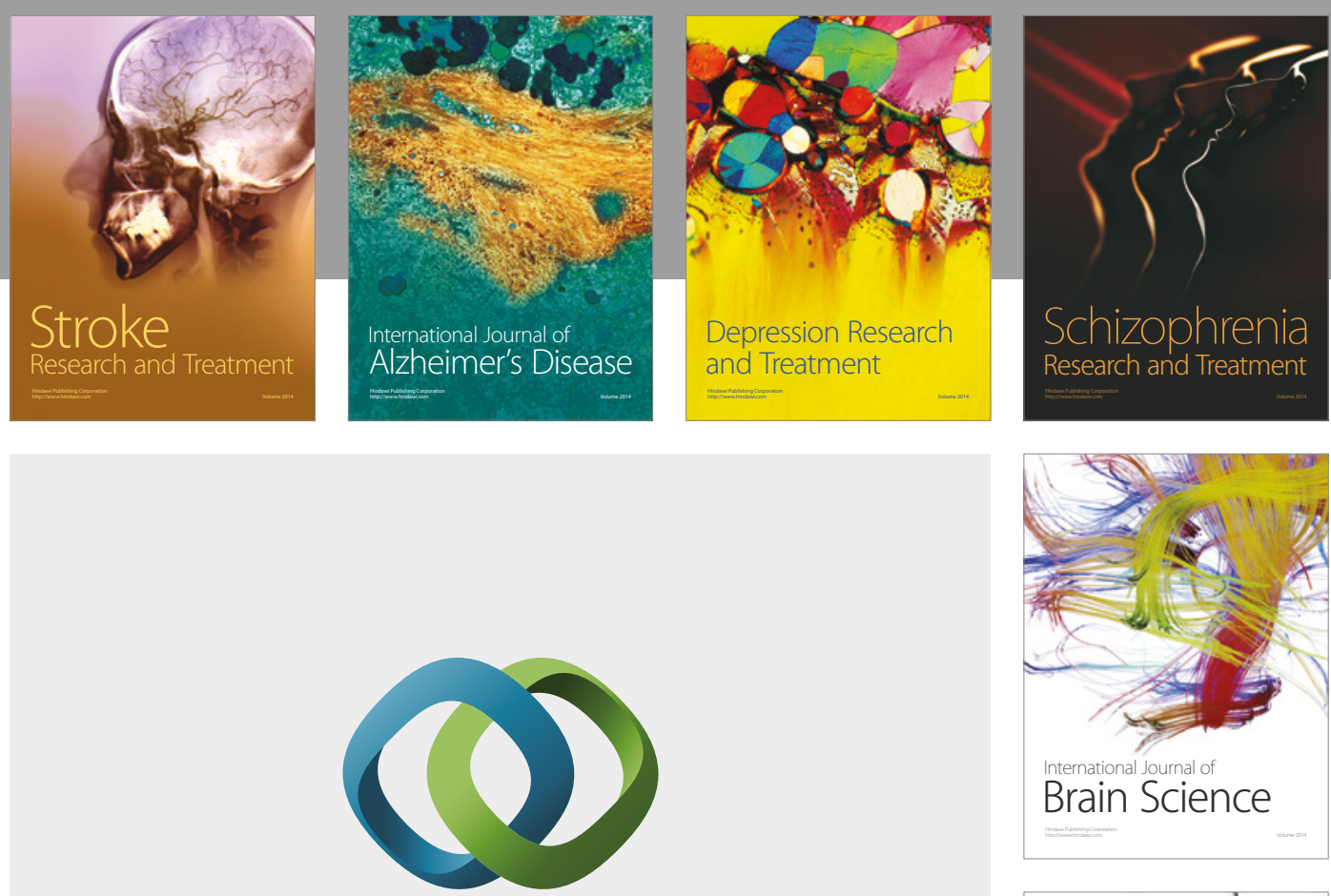

\section{Hindawi}

Submit your manuscripts at

https://www.hindawi.com
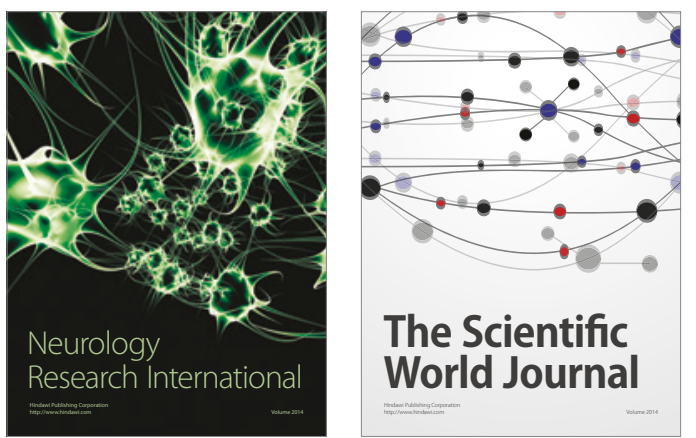

The Scientific World Journal

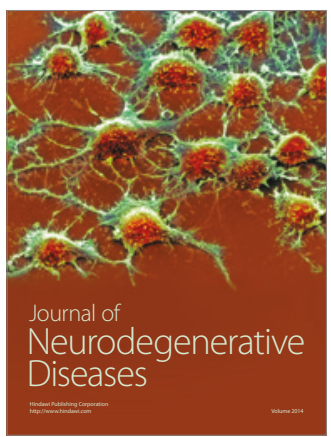

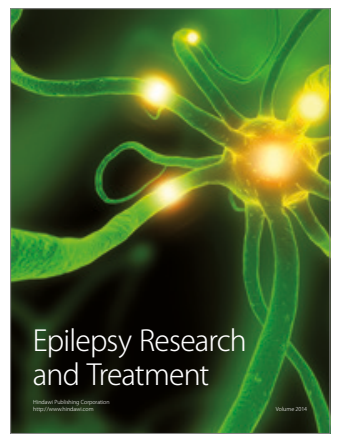

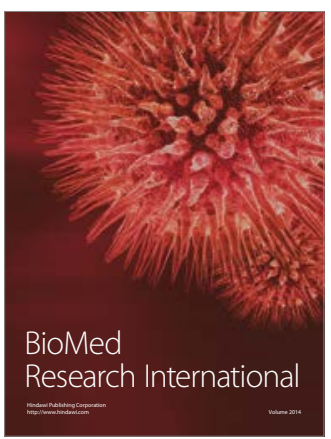

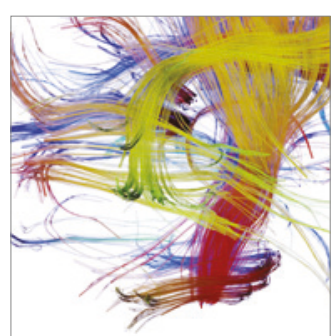

Brain Science

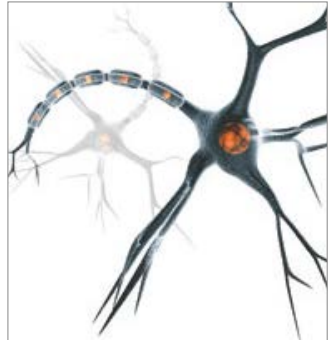

Neural Plasticity
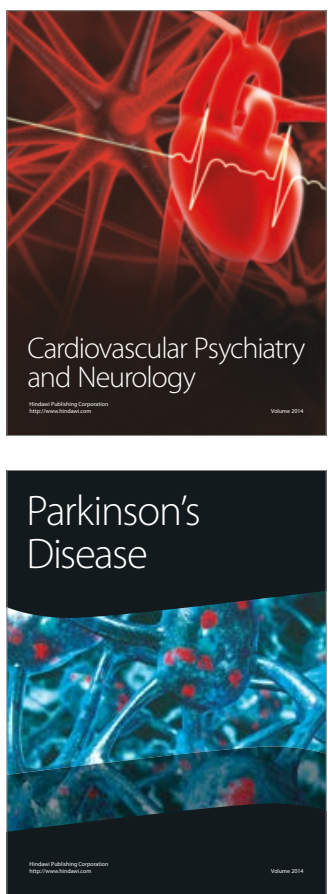